\title{
Competition between supersolid phases and magnetization plateaux in the frustrated easy-axis antiferromagnet on a triangular lattice
}

\author{
Luis Seabra ${ }^{1}$ and Nic Shannon ${ }^{1}$ \\ ${ }^{1}$ H. H. Wills Physics Laboratory, University of Bristol, Tyndall Av, BS8-1TL, UK.
}

(Dated: October 29, 2018)

\begin{abstract}
The majority of magnetic materials possess some degree of magnetic anisotropy, either at the level of a single ion, or in the exchange interactions between different magnetic ions. Where these exchange interactions are also frustrated, the competition between them and anisotropy can stabilize a wide variety of new phases in applied magnetic field. Motivated by the hexagonal delafossite $2 \mathrm{H}-$ $\mathrm{AgNiO}_{2}$, we study the Heisenberg antiferromagnet on a layered triangular lattice with competing first- and second-neighbour interactions and single-ion easy-axis anisotropy. Using a combination of classical Monte Carlo simulation, mean-field analysis, and Landau theory, we establish the magnetic phase diagram of this model as a function of temperature and magnetic field for a fixed ratio of exchange interactions, but with values of easy-axis anisotropy $D$ extending from the Heisenberg $(D=0)$ to the Ising $(D=\infty)$ limits. We uncover a rich variety of different magnetic phases. These include several phases which are magnetic supersolids (in the sense of Matsuda and Tstuneto or Liu and Fisher), one of which may already have been observed in $\mathrm{AgNiO}_{2}$. We explore how this particular supersolid arises through the closing of a gap in the spin-wave spectrum, and how it competes with rival collinear phases as the easy-axis anisotropy is increased. The finite temperature properties of this phase are found to be different from those of any previously studied magnetic supersolid.
\end{abstract}

PACS numbers: $67.80 . \mathrm{kb}, 75.10 .-\mathrm{b}, 75.10 . \mathrm{Hk}$

\section{INTRODUCTION}

The very real possibility of finding new quantum phases and excitations has made frustrated magnetism one of the most exciting and dynamic research areas in contemporary condensed matter physics ${ }^{12}$. Often, frustrated magnets have the potential to support many different ground states, and can be tuned at will between these states by applying a magnetic field. Relative to other approaches, such as chemical doping, this field control of phase transitions has the great advantage that it does not introduce new complications - such as disorder into the system. The advent of powerful new magnets, in some cases capable of reaching many hundreds of Tesla, has made it possible to study new magnetic phases and the (quantum) phase transitions between them in a wide range of materials for the first time. These high-field experiments have already yielded new insights into phenomena as diverse as Bose-Einstein condensation ${ }^{3}$, and the delicate interplay between magnetism and structural transitions in $\mathrm{Cr}$ spinels ${ }^{4}$.

Triangular lattice antiferromagnets have been central to our understanding of frustration since the pioneering work of Wannier ${ }^{5}$. A wide range of novel phases have first been proposed in the context of the triangular lattice, including the celebrated "resonating valence bond" (RVB) spin-liquid $\frac{617}{\text {, two-dimensional chiral }}$ phases $^{8}$, magnetisation plateaux ${ }^{910}$, and a collection of different multipolar states 11 14. Recently, spin-liquid behaviour has been established experimentally in a variety of triangular-lattice systems, including the quasi-2D organics $\kappa-(\mathrm{ET})_{2} \mathrm{Cu}_{2}(\mathrm{CN})_{3}{ }^{15}$ and $\mathrm{EtMe}_{3} \mathrm{Sb}\left[\mathrm{Pd}(\mathrm{dmit})_{2}\right]_{2}{ }^{16}$, thin films of ${ }^{3} \mathrm{He}$ [Ref. 17], and the layered pnic- tide $\mathrm{NiGa}_{2} \mathrm{As}_{4} 18$.

While the majority of this work assumes $S U(2)$ invariance - i.e. the absence of magnetic anisotropy - this is rarely true of real materials. Generically, these exhibit some degree of magnetic anisotropy, either at the level of a single ion, or in their exchange interactions ${ }^{19}$. It has been known for a long time that the interplay between magnetic field, frustration and magnetic anisotropy can lead to novel magnetic phases on the triangular lattice, even in simple classical models. The classical Heisenberg antiferromagnet with easy-axis anisotropy, first studied by Miyashita and Kawamura20121, exhibits a competition between the coplanarity favoured by magnetic interactions 8 , and collinear order driven by anisotropy and magnetic field ${ }^{22}$. More recent studies have highlighted successive Berezinskii-Kosterlitz-Thouless phase transitions as a function of temperature ${ }^{23 \mid 24}$ and exotic quantum effects at large single-ion anisotropy ${ }^{25}$.

One possible outcome of the competition between exchange interactions, magnetic anisotropy and magnetic field is a state which breaks both spin and lattice symmetries, exhibiting a finite (staggered) magnetisation perpendicular to the field, and broken translational symmetry. Matsuda and Tstunetc ${ }^{26}$ and Liu and Fisher ${ }^{27}$ argued, via a quantum lattice gas mapping, that such a mixed-symmetry phase could be an exact analogue of the supersolid phase proposed in ${ }^{4} \mathrm{He}$ [Ref. 28 30]. In the model considered by Liu and Fisher, a magnetic supersolid was found to interpolate between a collinear antiferromagnet and its high-field, canted, "spin-flop" state. This magnetic supersolid was argued to terminate in a finite-temperature tetracritical point, where collinear, supersolid, spin-flop and paramagnetic phases meet ${ }^{27}$. 
Recent, controversial, experiments on ${ }^{4} \mathrm{He}$ [Ref. 31. have lead to a renaissance of interest in supersolid phases ${ }^{32}$ and their magnetic analogues. Quantum Monte Carlo simulations now provide clear evidence for magnetic supersolids in $S=1 / 2$, easy-axis models on the triangular lattice 33 . In this case, following the original logic of Andree $\sqrt{30}$, the transition into a magnetic supersolid can be interpreted as a field-driven Bose-Einstein condensation of magnons within a magnetic solid - a collinear, magnetically ordered phase. Magnetic supersolids have also been associated with biconical phases 37138 .

Among triangular lattice magnets, $\mathrm{AgNiO}_{2}$ is of special interest. Combining metallicity and localised magnetism, its ground state is a collinear antiferromagnetic "stripe" phase ${ }^{39}$. When applying magnetic field a complicated and as of yet unexplained cascade of phase transitions is observed ${ }^{40}$. In a previous work we have argued that the low-field transition observed in $\mathrm{AgNiO}_{2}$ converts the collinear ground state into a novel state which is a magnetic supersolid in the sense of Matsuda and Tstuneto or Liu and Fisher ${ }^{41}$. In this case, the expected first-order spin-flop transition is replaced by a continuous transition into a magnetic supersolid. This occurs through the Bose-Einstein condensation of magnons with a finite momentum, leading to a novel magnetic supersolid distinct from any of the cases considered above.

In this paper we extend the analysis began in Ref. 41 to explore systematically how competing exchange interactions and easy-axis anisotropy provide a route to novel magnetic phases under applied field in, e.g., hexagonal delafossites. We consider explicitly the model used to fit low-energy magnetic excitations in $\mathrm{AgNiO}_{2}$, with competing first-neighbour $\left(J_{1}\right)$ and second-neighbour $\left(J_{2}\right)$ exchange interactions on a triangular lattice, small but finite inter-layer coupling $\left(J_{\perp}\right)$, and a varying degree of easy-axis anisotropy $D$ [Ref. 42. While there have been some theoretical studies on the interplay of furtherneighbour interactions and anisotropy on the triangular lattice $e^{43 \mid 44}$, the possibility of finding new phases in magnetic field remains largely unexplored.

In this paper we constrain the ratio between exchange parameters to values relevant to $\mathrm{AgNiO}_{2}$ and explore how the magnetic phase diagram evolves as a function of easy-axis anisotropy. The resulting behaviour is extremely rich, exhibiting a wide variety of magnetic supersolid and collinear plateau phases, (almost) all of which share a common four-site unit cell. Since our main interest is in finite-temperature phases and phase transitions, we consider the classical limit $S \rightarrow \infty$, which opens the problem to large-scale Monte Carlo simulation. This is complemented by zero-temperature mean-field theory, low-temperature spin-wave expansions, and Landau theory. Particular attention is paid to the novel magnetic supersolid phase introduced in Ref. 41. This is shown to have qualitatively different properties at finite temperature from any previously studied supersolid, and to be remarkably robust against changes in parameters.

The plan of the paper is as follows: in Section II we in-

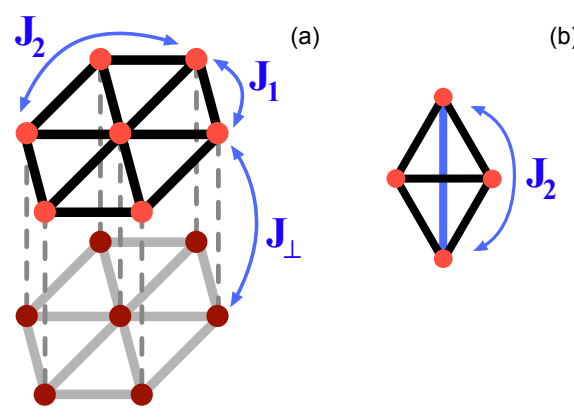

FIG. 1: (color online) (a) Layered triangular lattice considered in this paper, with first- and second- neighbour interactions $J_{1}$ and $J_{2}$ in each plane, coupled by interactions between perpendicular neighbours $J_{\perp}$. (b) Second-neighbour interactions transform the natural unit cell within the plane into a diamond plaquette.

troduce the model, discuss the choice of parameters and present mean-field arguments. We also briefly outline the numerical and analytical methods employed. In Sections. III [X] we explore the finite-temperature and finitefield behaviour of the model for representative values of easy-axis anisotropy, interpolating from the Heisenberg model $[D=0]$ to the Ising limit $[D=\infty]$. The main results of the paper are summarised in a corresponding series of phase diagrams : Fig. $6[D=0]$, Fig. 10 $[D=0.02]$, Fig. 13 [ $D=0.25]$, Fig. 24 [ $D=0.5]$, Fig. 26 $[D=0.65]$, Fig. $28[D=1.5]$ and Fig. $29[D=\infty]$. We summarise the key points of the results and discuss the remaining open questions in Section X. In Section XI we consider the relevance of these results to experiments on $\mathrm{AgNiO}_{2}$. We conclude in Section XII. Technical details of calculations of spin stiffness and low-temperature spin wave expansions are discussed in Appendix $\mathrm{A}$ and Appendix B.

\section{MODEL AND METHODS}

We consider the classical Heisenberg model on a layered triangular lattice

$$
\begin{aligned}
\mathcal{H}= & J_{1} \sum_{\langle i j\rangle_{1}} \mathbf{S}_{i} \cdot \mathbf{S}_{j}+J_{2} \sum_{\langle i j\rangle_{2}} \mathbf{S}_{i} \cdot \mathbf{S}_{j}+J_{\perp} \sum_{\langle i j\rangle_{\perp}} \mathbf{S}_{i} \cdot \mathbf{S}_{j} \\
& -D \sum_{i}\left(S_{i}^{z}\right)^{2}-h \sum_{i} S_{i}^{z},
\end{aligned}
$$

with competing antiferromagnetic interactions $J_{1}>0$ and $J_{2}>0$ on the first and second neighbour bonds of a triangular lattice plane [Fig. 1]. Neighbouring planes are coupled by an interlayer exchange $J_{\perp}$. Easy-axis anisotropy is characterised by a single-ion term $D$ and the magnetic field $h$ is assumed to lie along the $z$ axis. We set $|\mathbf{S}|=S=1$ throughout, and all energy scales including field $h$ and temperature $T$ are measured in units of $J_{1}$ hereafter.

We fix first-neighbour interactions to be $J_{1} \equiv 1$ and set interlayer exchange $J_{\perp}=-0.15$. This leaves three distinct 
free parameters: second-neighbour coupling $J_{2}$, easy-axis anisotropy $D$ and external field $h$. We note that the $h=0, J_{\perp}=0, D \rightarrow \infty$ limit of Eq. (1) corresponds to the frustrated Ising model previously studied in e.g. Refs. 45 and 46.

Second-neighbour interactions change the "natural" description of the lattice from edge-sharing triangles to edge-sharing four-site "diamond" plaquettes, which can be thought of as a $2 \mathrm{D}$ projection of an irregular tetrahedron. Thus, at a mean-field level, the exchange terms of Eq. (1) can be rewritten in terms of the total magnetisation of each plaquette

$$
\mathcal{H}_{e x}=\frac{J_{1}+J_{2}}{4} \sum_{\diamond}^{N / 4}\left|\mathbf{S}_{A}+\mathbf{S}_{B}+\mathbf{S}_{C}+\mathbf{S}_{D}\right|^{2}-4 .
$$

This allows an interesting parallel with the physics of tetrahedra embedded in a pyrochlore lattice in a bilinearbiquadratic model $\sqrt{4 \mid 47 / 48}$, explored further below.

The application of a magnetic field reveals a cascade of new states, summarised in Fig. 2, These consist of :

(a) A two-sublattice, collinear antiferromagnetic "stripe" state [Fig. 2(a)], which is the ground state in the absence of magnetic field, and which breaks both the translational and rotation symmetries of the lattice.

(b) A two-sublattice canted "spin-flop" state [Fig. 2(d)] which breaks both the rotational symmetries of the lattice, and spin-rotation symmetry in the $S^{x}-S^{y}$ plane.

(c) A novel magnetic supersolid state [Fig. 2(e)] with a four-site unit cell which breaks both the translational and rotational symmetries of the lattice, and spinrotational symmetry in the $S^{x}-S^{y}$ plane

(d) A three-sublattice, $m=1 / 3$ magnetisation plateau [Fig. 2(b)] which breaks the translation symmetries of the lattice.

(e) A four-sublattice, 2:1:1 canted magnetic supersolid state [Fig. 2(f)], which breaks both the rotational symmetries of the lattice and spin-rotational symmetry in the $S^{x}-S^{y}$ plane

(f) A four-sublattice, $m=1 / 2$ magnetisation plateau [Fig. 2(c)] which breaks both the translational and rotational symmetries of the lattice.

(g) A four-sublattice, 3:1 canted magnetic supersolid state [Fig. 2(g)], which breaks both the rotational symmetries of the lattice and spin-rotational symmetry in the $S^{x}-S^{y}$ plane

Each of these phase will be described in more detail below. In what follows we shall focus in particular on the novel magnetic supersolid state [Fig. 2(c)], previously introduced in Ref. 41 as an explanation for the low-field transition observed in $\mathrm{AgNiO}_{2}{ }^{40}$.
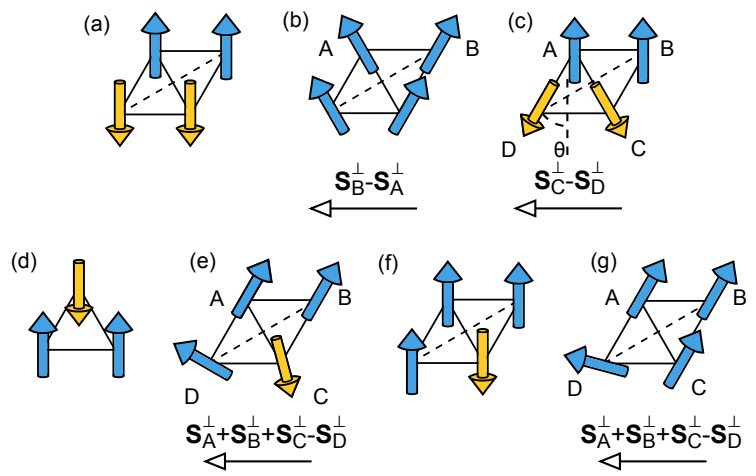

FIG. 2: (color online) Cartoons of different three- and foursublattice ground states found at mean-field level as a function of field. (a) Collinear stripe phase, (b) "Spin-flopped" version of the stripe phase. (c) novel magnetic supersolid state, the main focus of this paper. (d) $m=1 / 3$ magnetisation plateau. (e) 2:1:1 canted phase. (f) $m=1 / 2$ magnetisation plateau. (g) 3:1 canted phase. The staggered moment in the $S^{x}-S^{y}$ plane is shown as a vector for states (b), (c), (e) and (g).

To get a first idea of how these phases fit together, let us first consider the phase diagram in the absence of field, for a range of $J_{2}$ and $D$, within zero-temperature mean field theory [Fig. 3. For vanishing magnetic anisotropy $(D=0)$ and $J_{2}<1 / 8$, we find the well-known coplanar three-sublattice $120^{\circ}$ ground state ${ }^{8}$. For $1 / 8<J_{2}<1$ this gives way to a set of classically-degenerate twosublattice antiferromagnetic configurations. These are locked together, by therma ${ }^{49}$ or quantum fluctuations, to form collinear "stripe" order [Fig,4(a)]50|51. For $J_{2}>1$ the ground state is a one-dimensional coplanar spiral with wave number $q=(2 / \sqrt{3}) \cos ^{-1}\left[-\left(J_{1}+J_{2}\right) /\left(2 J_{2}\right)\right]$ [Ref. 49150. The inclusion of easy-axis anisotropy further stabilizes the collinear stripe state, and converts the $120^{\circ}$ state and coplanar spirals into a three-sublattice coplanar fan ${ }^{21}$, and a one-dimensional helicoidal phase, respectively [Fig. 3 .

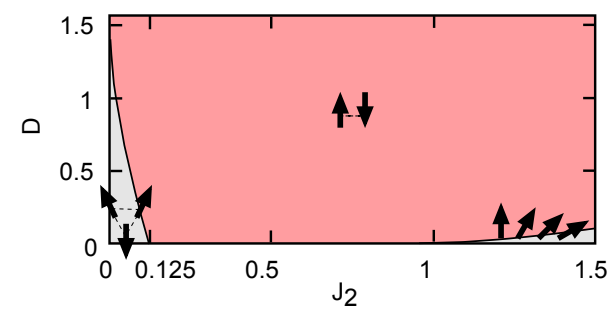

FIG. 3: (color online) Zero field $J_{2}-D$ phase diagram for $J_{1}=1$ and $J_{\perp}=-0.15$ from zero temperature mean-field theory. The model supports three different ground states : a three-sublattice coplanar state, a two-sublattice collinear "stripe" antiferromagnet and an incommensurate helicoidal phase.

Now let us consider how this zero-temperature phase diagram evolves with field. A reasonable expectation would be that, for sufficiently large field, the collinear stripe state [Fig. 2(a)] would undergo a first-order 


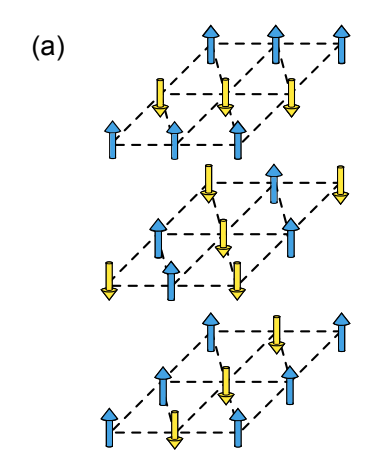

(b)

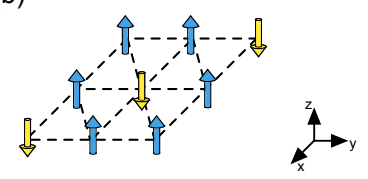

(e)
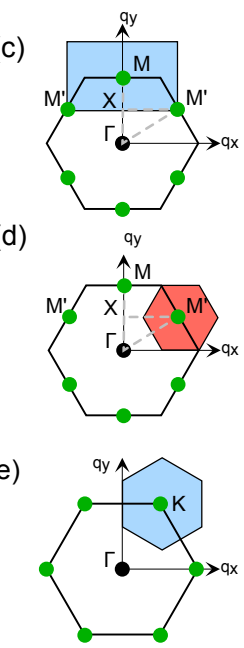

FIG. 4: (color online) (a) Collinear stripe phase favoured by second-neighbour interactions, showing the three possible stripe orientations. (b) Three-sublattice $m=1 / 3$ plateau state with two spins up and one down. (c) First Brillouin zone in $q_{x}-q_{y}$ plane showing momentum vectors $M$ and $M^{\prime}$ associated with the stripe phases. The blue rectangle shows the first magnetic Brillouin zone for the stripe phase with ordering vector $M$ (top cartoon). (d) The red hexagon shows the first magnetic Brillouin zone for a generic four-sublattice state centred on $M^{\prime}$. (e) Ordering vectors $K$ of the $m=1 / 3$ plateau state and related first magnetic Brillouin zone, shaded blue.

"spin-flop" transition into a two-sublattice canted state [Fig. 2(b)]. However this is not what happens. The first new phase encountered as magnetic field is increased, within mean field theory, is shown as a function of $J_{2}$ and $D$ in Fig. 5 At small $J_{2}$, we find the familiar collinear $m=1 / 3$ magnetisation plateau [Fig. 4(c)] and for large $D$, moderate $J_{2}$, a collinear $m=1 / 2$ magnetisation plateau [Fig. 2(f)]; for large $J_{2}$ and small $D$ the helicoidal phase interpolates all the way to saturation. However, away from the margins of the phase diagram, a novel four-sublattice, partially canted phase [Fig. 2(c)] completely dominates, displacing the more conventional spin-flop phase. This partially canted state is a magnetic supersolid phase, according to the definition of Matsuda and Tsunetd ${ }^{26}$ or Liu and Fisher ${ }^{[27}$. While the stripe state breaks only discrete lattice symmetries, and the canted spin-flop state only continuous spin-rotational ones, the partially canted supersolid breaks both. The reasons for the emergence of this unusual - and very robust - new state form the main focus of this work.

For the purposes of this paper we choose to fix the nearest-neighbour interaction $J_{2}=0.15$ and vary the anisotropy strength, selecting values along the vertical dashed line in Fig. 3. This line includes the parameters $J_{2}=0.15 J_{1}$ and $D=2 / 3 J_{1}$ relevant to the magnet $\mathrm{AgNiO}_{2}$ [Ref. 42$]$ discussed above $\mathrm{H}^{71}$. Moreover, since the $J_{2}=0.15$ line lies close to several phase boundaries in $J_{2}-D$ space, it opens the possibility of field-driven competition between different phases.

The main results of this paper are a set of finite-

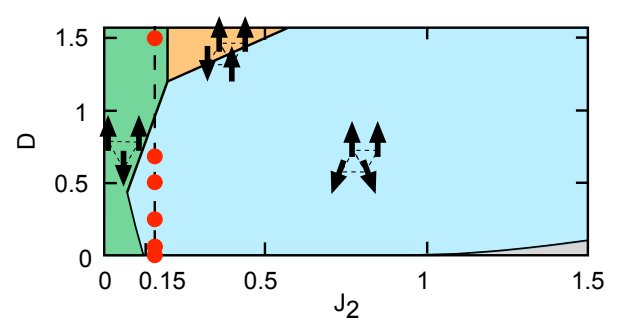

FIG. 5: (color online) Finite-field $J_{2}-D$ phase diagram for $J_{1}=1$ and $J_{\perp}=-0.15$, showing the first mean-field instability with applied field. Three different states are found : $m=1 / 3$ and $m=1 / 2$ magnetisation plateaux and magnetic supersolid state. Red dots along dashed vertical line show studied anisotropy values.

temperature, finite-field phase diagrams for Eq. (1) obtained using classical Monte Carlo (MC) simulation. Because of the strong magnetic frustration and magnetic anisotropy, and a large number of first-order phase transitions, MC simulations of this model are very challenging. To overcome severe slowing down in simulation dynamics we employ a parallel tempering $\mathrm{MC}$ scheme ${ }^{52}$, which combines local Metropolis updates with parallel simulation of multiple replicas of the system at different temperatures. The exchange of replicas at different temperatures allows the system to avoid local free energy minima traps. To further reduce the autocorrelation between spin configurations, especially in the equilibration phase, every local-update sweep is followed by successive over-relaxation sweeps 53 . These are entirely deterministic, and comprise the reflection of each spin in turn in the plane formed by the $S^{z}$ easy axis and the local field from its neighbouring spins. This over-relaxation scheme is a micro-canonical update, and reversible, and therefore the global Markov chain for parallel tempering and over-relaxation will also obey detailed balance.

Simulations of from 48 to 160 replicas were performed for rhombohedral clusters of $3 L \times 3 L \times L=9 L^{3}$ spins with periodic boundary conditions, where $L=4,6,8,10$ is the cluster thickness, i.e. counts the number of triangular lattice planes. This cluster geometry was chosen to reflect the higher correlation length in the $\hat{\mathbf{x}}-\hat{\mathbf{y}}$ plane relative to the $\hat{\mathbf{z}}$ axis for $J_{\perp} / J_{1} \approx 0.1$. Typical simulations involved $4 \times 10^{6}$ steps, half of which were discarded for thermalization. Each step consisted of one local-update sweep of the lattice followed by two over-relaxation sweeps, with replicas at different temperatures exchanged every 10 steps. Typically, simulations were started from initial random configurations. Nevertheless, the competition between three and four-sublattice states leads to strongly first order transitions involving large internal energy discontinuities at low temperature, difficult to overcome even with parallel tempering. In order to overcome this, the results in this region were obtained through careful comparison between several runs with different initial configurations. Some runs were initialised with fully ordered states and others with mixed phase configurations be- 

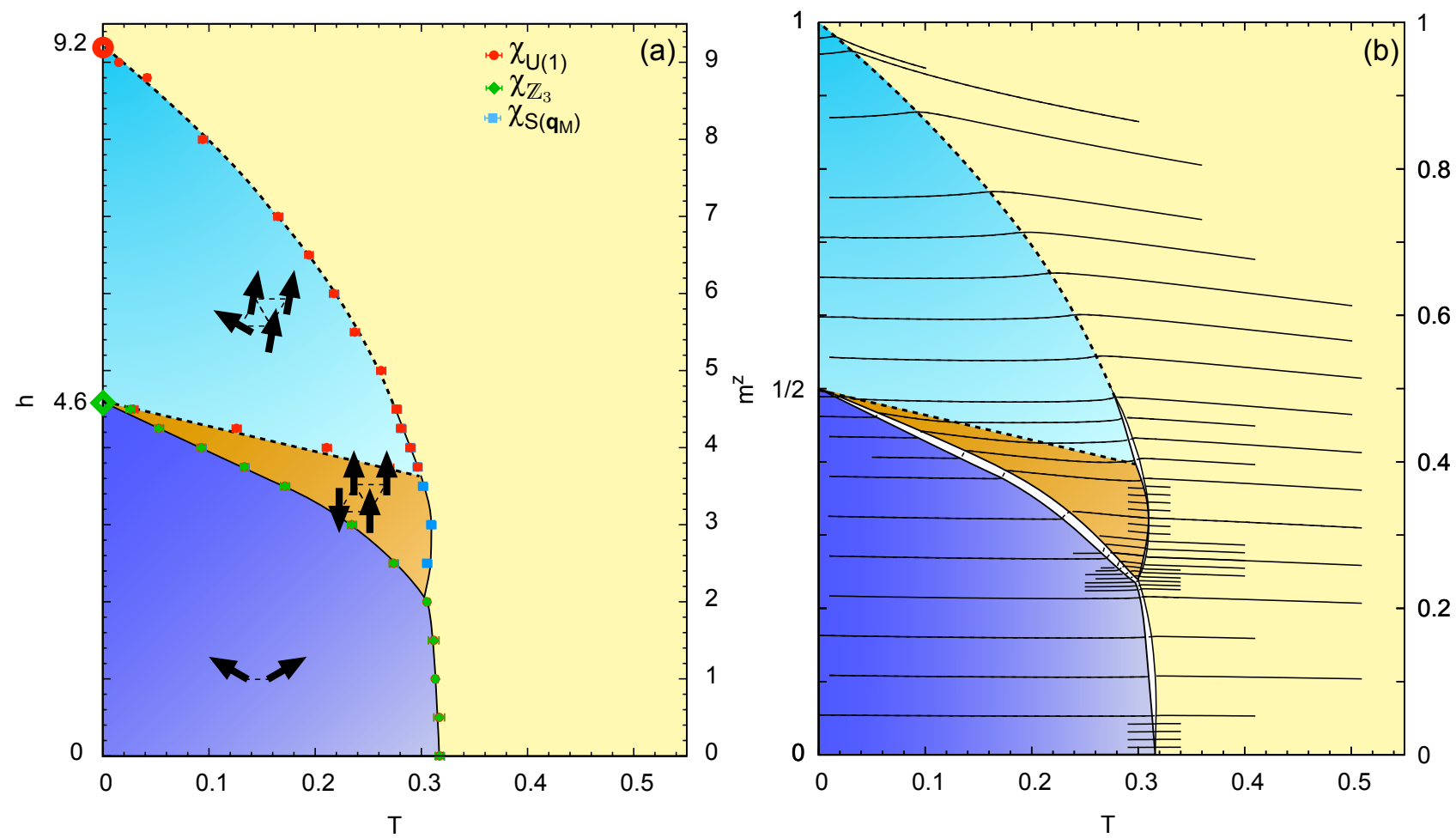

FIG. 6: (color online) Magnetic phases of a layered triangular-lattice antiferromagnet with exchange interactions $J_{1}=1, J_{2}=0.15$, $J_{\perp}=-0.15$ and easy-axis anisotropy $D=0$. (a) Phase diagram as a function of temperature and magnetic field. Open symbols on the h-axis show transitions obtained in mean-field theory. Phase boundaries at finite temperature are obtained from Monte Carlo simulation for a cluster of $24 \times 24 \times 8$ spins, and determined by peaks in the relevant order parameter susceptibilities. All phase transitions are firstorder, except where shown with a dashed line. All phases share a 4-sublattice unit-cell. (b) Phase diagram as a function of temperature and magnetization. Solid lines running left-right show cuts at constant magnetic field $h$ taken from simulations. The coexistence regions associated with first order phase transitions are coloured white.

tween both types of order.

These simulations are complemented by analytic calculations at low temperature. The $T=0$ ground state of the model can be calculated within mean-field theory for 2, 3 and 4 sublattice states. This provides a direct check on the $T=0$ limit of the phase transitions found in simulations. The Gaussian fluctuations about these meanfield states are calculated using the low-temperature spinwave technique outlined in Appendix A. We use this approach to calculate magnetisation curves at finite field, and to parametrize a Landau theory for continuous phase transitions. These results are compared with phase boundaries obtained from simulation in the phase diagrams below.

\section{HEISENBERG LIMIT, $D=0$}

The two natural limits of the Hamiltonian Eq. (1) are the Heisenberg $(D=0)$ and Ising $(D=\infty)$ models. We consider a range of $D$ interpolating between these, beginning with a pure Heisenberg model. For $D=0$, the phase diagram is dictated by the order-from-disorder selection of a small number of magnetically ordered phases from a disordered manifold of competing ground states. In the absence of magnetic field, like previous authors4950, we find that fluctuations favour a collinear antiferromagnetic "stripe" ground state. Once magnetic field is applied, these stripes cant. At a field of $h=4\left(J_{1}+J_{2}\right)=4.6$ $\left(T=0^{+}\right)$, there is a first order transition from the 2sublattice canted state into a 4 -sublattice collinear halfmagnetization plateau state. As field is increased further, this plateau undergoes a continuous phase transition into a $3: 1$ canted state, which interpolates to saturation. Finally the system saturates at a field of $h=9.2(T=0)$. These results are summarised in Fig. 6, and described in more detail below.

In the absence of magnetic field $(h=0)$ the zerotemperature mean-field ground state of Eq. (1) is a manifold of states built of two decoupled Néel sublattices [Fig. 7(a)]. Thermal fluctuations select the collinear stripe phase [Fig. 7(b)] which maximizes the entropy accessible to spin wave excitations. This stripe state breaks both $O(3)$ spin-rotation symmetry and the (discrete) rotation symmetry of the lattice - the stripes can be orientated in three different ways [Fig. 4(a)]. To study the finite temperature transition into this state, we therefore introduce a complex order parameter $\psi=\psi_{1}+i \psi_{2}$ based on a two-dimensional irreducible representation of 


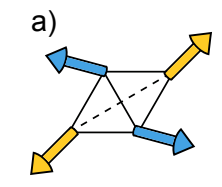

c)

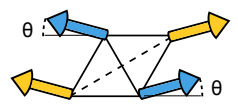

b)

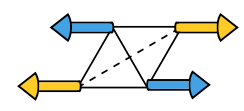

d)

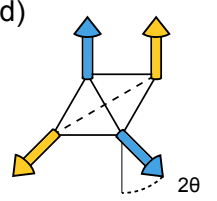

FIG. 7: (color online) Structure of the ground state of the $J_{1}-J_{2}$ Heisenberg model on a triangular lattice for $J_{2} / J_{1}>1 / 8$. (a) Degenerate manifold of states for $T=0, h=0$, built of two independent Néel sublattices. (b) Collinear antiferromagnetic stripe phase selected by thermal fluctuations $(h=0)$. (c) "Spin flop" phase formed by direct canting of antiferromagnetic stripes in applied magnetic field. (d) Supersolid phase formed from the same two canted sublattices.

the $C_{3} \cong \mathbb{Z}_{3}$ lattice rotation group

$$
\begin{aligned}
& \psi_{1}=\frac{1}{\sqrt{6} N} \sum_{i} 2 \mathbf{S}_{i} \cdot \mathbf{S}_{i+\delta_{1}}-\mathbf{S}_{i} \cdot \mathbf{S}_{i+\delta_{2}}-\mathbf{S}_{i} \cdot \mathbf{S}_{i-\delta_{1}-\delta_{2}}, \\
& \psi_{2}=-\frac{1}{\sqrt{2} N} \sum_{i} \mathbf{S}_{i} \cdot \mathbf{S}_{i+\delta_{2}}-\mathbf{S}_{i} \cdot \mathbf{S}_{i-\delta_{1}-\delta_{2}},
\end{aligned}
$$

Here the primitive vectors of the triangular lattice are $\delta_{1}=(1,0), \delta_{2}=(1 / 2, \sqrt{3} / 2)$. Since parallel tempering effectively restores this lattice symmetry, we measure the magnitude of the order parameter

$$
O_{\mathbb{Z}_{3}}=\left\langle\sqrt{\left|\psi_{1}\right|^{2}+\left|\psi_{2}\right|^{2}}\right\rangle,
$$

which is normalised to $4 / \sqrt{6}$ for perfect stripe order. In Fig. 8 we present MC simulation results for the finite temperature transition from the paramagnetic into the collinear antiferromagnetic state. The transition is found to be first order, as expected for a 3-state Potts model in 3D (see e.g. Ref. 49).

Applying a magnetic field causes the spins in each Néel sublattice to cant. This can happen in two distinct ways. Both stripes can cant equally, to give the spin flop state shown in Fig. 7(c). Alternatively, the sublattices can rotate so that half of the spins are aligned with the magnetic field, while the others are canted away from it the supersolid state shown in Fig. 7(d). These two states are degenerate, but thermal fluctuations favour the spinflop state over the supersolid, since it has a higher density of spin wave excitations at low energies.

In the spin-flop phase all spins have equal $S^{z}$ components, and the lattice symmetries are broken only by spin components in the $S^{x}-S^{y}$ plane. We therefore modify Eq. (3) to read

$$
\begin{aligned}
& \psi_{1}^{\perp}=\frac{1}{\sqrt{6} N} \sum_{i} 2 \mathbf{S}_{i}^{\perp} \cdot \mathbf{S}_{i+\delta_{1}}^{\perp}-\mathbf{S}_{i}^{\perp} \cdot \mathbf{S}_{i+\delta_{2}}^{\perp}-\mathbf{S}_{i}^{\perp} \cdot \mathbf{S}_{i-\delta_{1}-\delta_{2}}^{\perp}, \\
& \psi_{2}^{\perp}=-\frac{1}{\sqrt{2} N} \sum_{i} \mathbf{S}_{i}^{\perp} \cdot \mathbf{S}_{i+\delta_{2}}^{\perp}-\mathbf{S}_{i}^{\perp} \cdot \mathbf{S}_{i-\delta_{1}-\delta_{2}}^{\perp},
\end{aligned}
$$
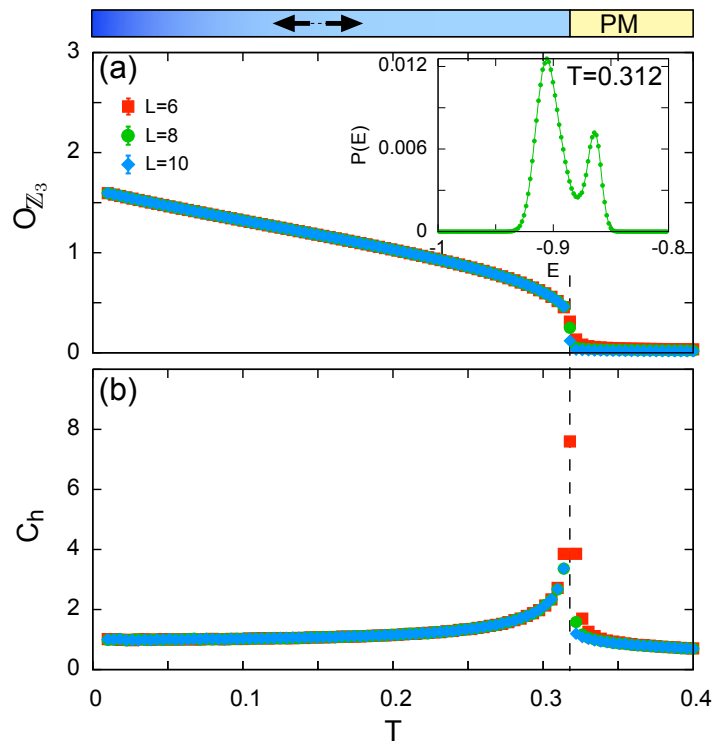

FIG. 8: (color online) Phase transition at $D=0, h=0$ from the paramagnet into the isotropic collinear antiferromagnetic stripe state. (a) Stripe order parameter from broken $\mathbb{Z}_{3}$ lattice symmetry, Eq. (3), and (b) heat capacity. The first-order behaviour is clear in the bimodal distribution of the energy histograms close to the critical temperature, inset to (a).

where $\mathbf{S}_{i}^{\perp}=\left(S_{i}^{x}, S_{i}^{y}\right)$, and consider the order parameter

$$
O_{\mathbb{Z}_{3}}^{\perp}=\left\langle\sqrt{\left|\psi_{1}^{\perp}\right|^{2}+\left|\psi_{2}^{\perp}\right|^{2}}\right\rangle .
$$

The spin-flop phase also separately breaks spin-rotation symmetry in the $S^{x}-S^{y}$ plane. This can be determined using an order parameter for the staggered in-plane magnetization

$$
O_{U(1)}=\frac{1}{N} \sum_{\diamond}^{N / 4}\left|\mathbf{S}_{B}^{\perp}-\mathbf{S}_{A}^{\perp}\right|
$$

where $A$ and $B$ label the two sublattices of the spin-flop state, as illustrated in Fig. 2 (b). The breaking of spinrotation symmetry implies the existence of a finite spin stiffness $\rho_{S}$, as defined in Appendix A.

At higher values of magnetic field, thermal fluctuations select another new ordered state from a large set of competing alternatives. This is a collinear state with a 4-site unit cell in which three spins point "up" and one "down" - commonly referred to as a half-magnetization $(m=1 / 2)$ plateau [Fig. 2(f)]. In the limit of vanishing temperature, this first occurs at a magnetic field value of $h=4\left(J_{1}+J_{2}\right)=4.6$. This collinear phase breaks translational symmetries, with associated ordering vectors $\left\{\mathbf{q}_{\mathrm{M}}\right\}$, cf. Fig. 4. The $S^{z}-S^{z}$ component of the static spin structure factor at these wave vectors

$$
\mathcal{S}^{z z}\left(\mathbf{q}_{\mathrm{M}}\right)=\left\langle\sum_{\left\{q_{M}\right\}}\left|\frac{1}{N} \sum_{i} S_{i}^{z} \mathrm{e}^{-i \mathbf{q}_{\mathrm{M}} \cdot \mathbf{r}_{i}}\right|^{2}\right\rangle,
$$



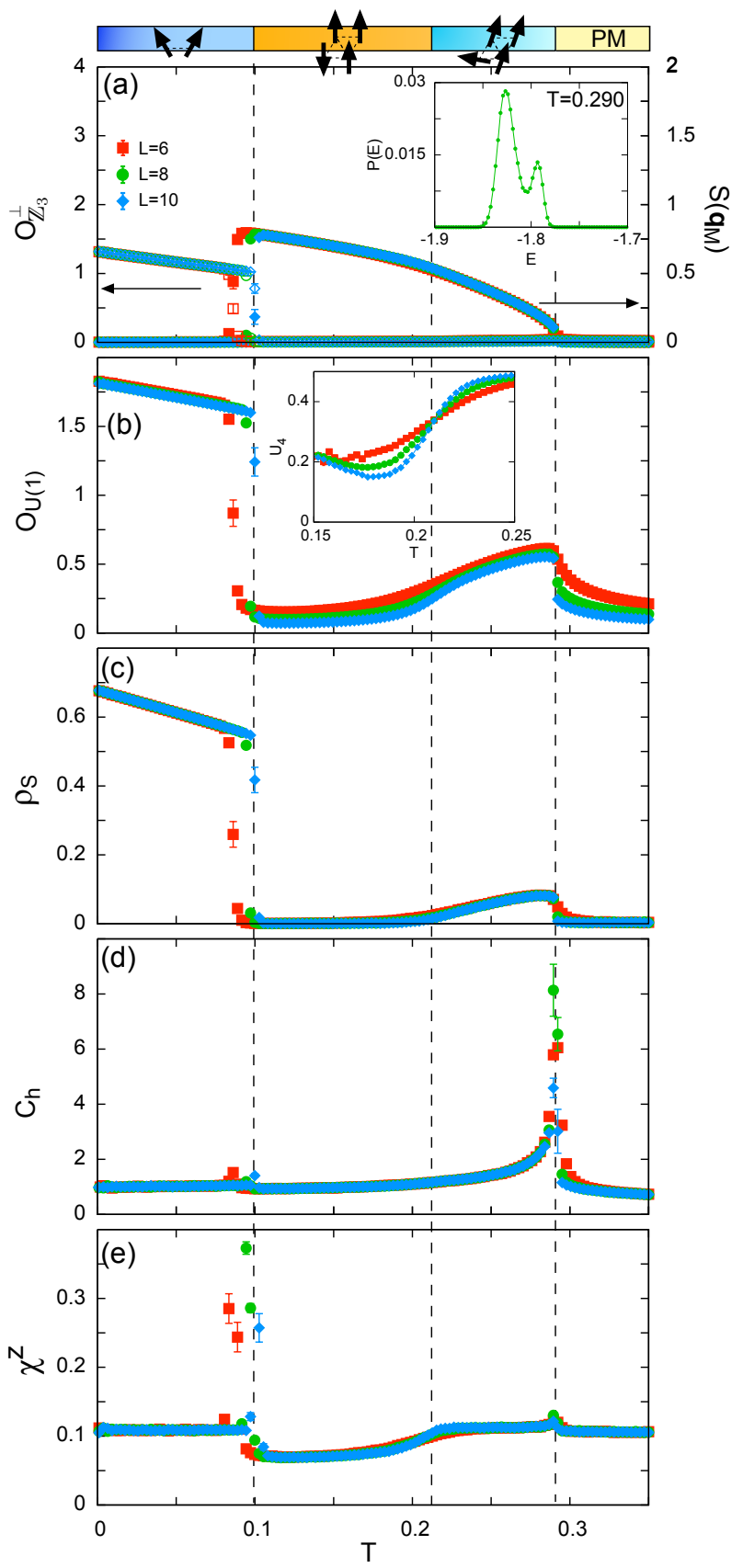

FIG. 9: (color online) Selection of simulation results for $D=0$, $h=4.0$, spanning the canted "spin-flop" phase, collinear $m=1 / 2$ plateau, 3:1 canted state, and high temperature paramagnet. (a) $\mathbb{Z}_{3}$ order parameter $O_{\mathbb{Z}_{3}}$ associated with broken lattice rotation symmetry in the spin flop state [Eq. [3] ], and spin structure factor $\mathcal{S}^{z z}\left(\mathbf{q M}_{\mathrm{M}}\right)$ associated with broken translational symmetry in the 3:1 canted and collinear $m=1 / 2$ plateau states [Eq. [6]]; (b) in-plane staggered magnetization $O_{U(1)}$ [Eq. [5]]; (c) spin stiffness $\rho_{S} ;$ (d) heat capacity $C_{h}$; (e) magnetic susceptibility $\chi$. The inset to (a) shows the energy distribution at the transition from paramagnet to $3: 1$ canted phase. The inset to (b) shows the crossing of the Binder cumulants associated with $O_{U(1)}$ at the transition from the 3:1 canted phase to the collinear $m=1 / 2$ plateau state.

acts as (the square of) an order parameter for this state. Since the state is collinear, and aligned with the magnetic field, its spin stiffness $\rho_{S} \equiv 0$. We note that, since the "down" spin is unique within the 4-site unit cell, this collinear state also breaks the permutation symmetries of the bonds within the unit cell. It is therefore possible to define a complementary $\mathbb{Z}_{4}$ order parameter for this state, based on the irreps of the relevant permutation group. For present purposes, however, the combination of a vanishing $\rho_{S}$ and a finite $\mathcal{S}^{z z}\left(\mathbf{q}_{\mathrm{M}}\right)$ are sufficient to identify the state.

The catalogue of ordered phases for $D=0$ is completed by a $3: 1$ canted phase [Fig. 2 (g)], which is connected to collinear $m=1 / 2$ plateau by a continuous phase transition as a function of magnetic field. All spin wave modes are gapped in the collinear plateau state. With increasing magnetic field, the gap associated with one of the zone-centre spin waves closes. Since this spin wave is a transverse excitation, it converts the collinear state into one with a staggered magnetization in the $S^{x}-S^{y}$ plane, and a finite spin stiffness. And, since, it is a zone-centre mode, the 3:1 canted phase inherits the broken translational symmetry of the $m=1 / 2$ plateau. The resulting state is therefore a supersolid, in the sense of Matsuda and Tstuneto or Liu and Fisher, breaking both translational and spin-rotational symmetries.

In Fig. 9 we show a selection of finite temperature simulation results for $h=4.0$, a cut through the $T-h$ plane which spans all three ordered states. The transition from the high temperature paramagnet to the $3: 1$ canted phase at $T=0.290(3)$ can be seen in the temperature dependence of $\mathcal{S}^{z z}\left(\mathbf{q}_{\mathrm{M}}\right)$ [Fig. 9(a)], staggered magnetization $O_{U(1)}\left[\right.$ Fig. 9(b)], spin stiffness $\rho_{S}$ [Fig. 9(c)], and a sharp feature in $C_{h}$ [Fig. 9(d)]. While symmetry permits a second-order phase transition, the phase transition observed in simulation for this value of $h$ is first-order, as shown by the energy histograms in the inset to Fig. 9 (a). The transition from $3: 1$ canted phase to $m=1 / 2$ plateau is distinguished by a collapse of the spin stiffness [Fig. 9(c)], $O_{U(1)}[\mathrm{Fig} .9$ (b)] and a suppression of the magnetic susceptibility $\chi$ [Fig. 9(e)]. The transition is continuous, with the Binder cumulant for the two-component $U(1)$ order parameter $U_{4}=1-\frac{\left\langle O_{U(1)}^{4}\right\rangle}{2\left\langle O_{U(1)}^{2}\right\rangle^{2}}$ crossing at a single temperature $T=0.210(3)$ for a range of system sizes [inset to Fig. 9(b)]. A strong finite size dependence of this Binder cumulant is observed for all observed continuous phase transitions. These finite-size corrections are especially large in ordered phases (e.g. plateau) where the relevant $U(1)$ order parameter vanishes in the thermodynamic limit.

Finally, the abrupt, first-order transition from the $m=1 / 2$ plateau to the spin flop phase at $T=0.100(3)$ is visible in the sudden onset of $O_{\mathbb{Z}_{3}}$ [Fig. 9(a)], $O_{U(1)}$ [Fig. 9(b)] and spin stiffness [Fig. 9(c)]. Here a continuous phase transition can be ruled out on symmetry grounds.

The magnetic phase diagram can also be plotted as a function of temperature and magnetisation [Fig. 6(b)]. In this case a discontinuity in the magnetisation as a function of temperature or magnetic field implies a first-order 

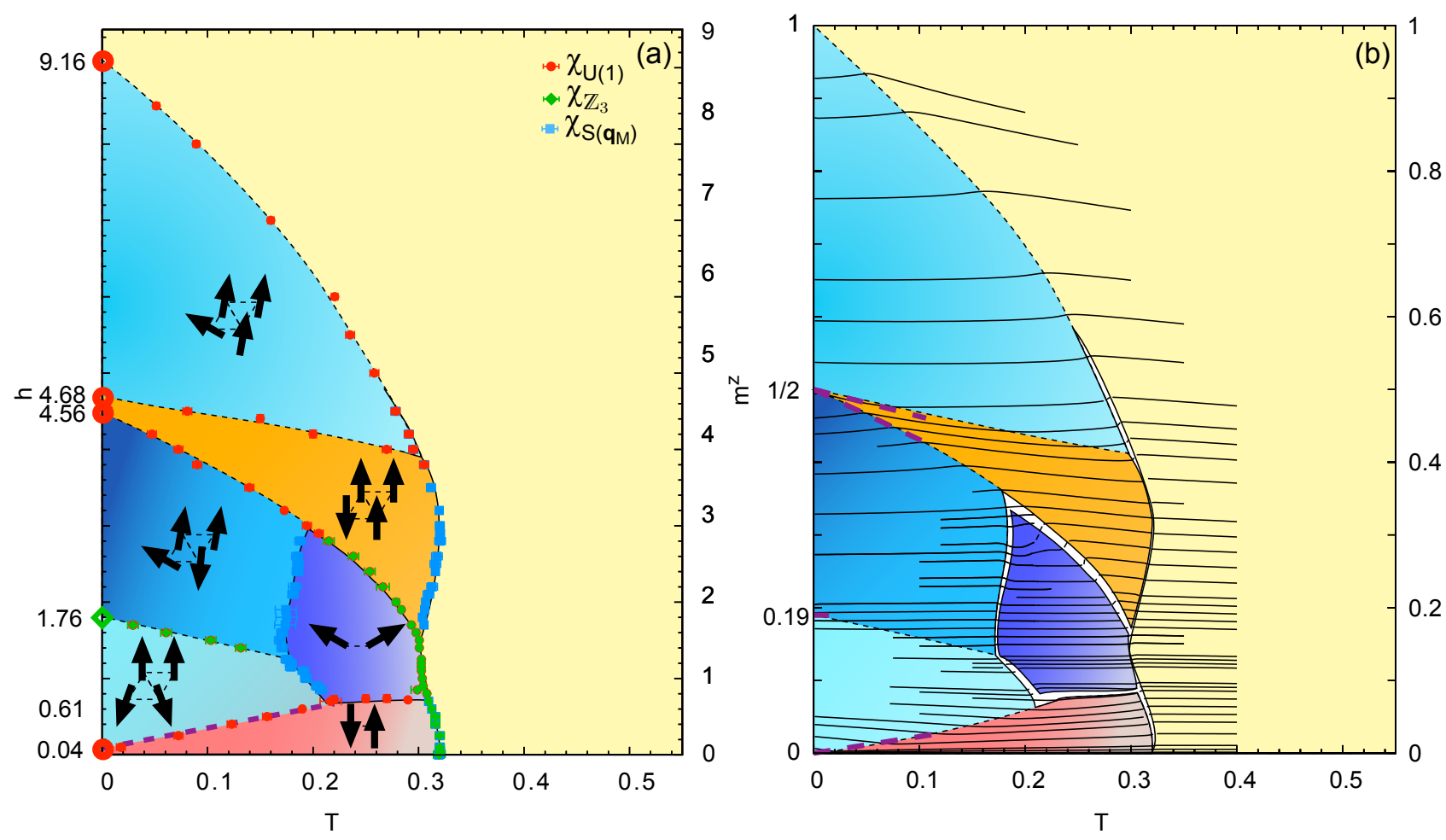

FIG. 10: (color online) Magnetic phases of a layered triangular-lattice antiferromagnet with exchange interactions $J_{1}=1, J_{2}=0.15$, $J_{\perp}=-0.15$ and easy-axis anisotropy $D=0.02$. (a) Phase diagram as a function of temperature and magnetic field. Open symbols on the h-axis show transitions obtained in mean-field theory. Phase boundaries at finite temperature are obtained from Monte Carlo simulation for a cluster of $24 \times 24 \times 8$ spins, and determined by peaks in the relevant order parameter susceptibilities. All phase transitions are first-order, except where shown with a dashed line. Thick purple dashed line is obtained through a Landau expansion for the supersolid transition. (b) Phase diagram as a function of temperature and magnetization. Solid lines running left-right show cuts at constant magnetic field $h$ taken from simulations. Thick purple dashed lines show phase boundaries obtained through a low-T expansion. Finite anisotropy lifts $T=0$ degeneracy in favour of the stripe and supersolid phases, relegating the spin-flop to finite-temperature only.

phase transition, and leads to regions of phase coexistence in the $T-m$ plane, coloured white in Fig. 6(b). The transition from the paramagnet to the $m=1 / 2$ plateau is thus seen to be first order, although with a very small magnetisation jump. The phase transition from the spinflop phase to the paramagnet is also first order for $h>0$.

The transition between the $m=1 / 2$ plateau and the $3: 1$ canted state is continuous, as is the finite-temperature transition from the 3:1 canted state to the paramagnet, for $h \gtrsim 5(m \gtrsim 0.5)$. The $3: 1$ canted phase finally saturates at zero temperature, at a field $h_{\mathrm{SAT}}=9.2$.

It is interesting to note that the same succession of phases in magnetic field occurs in a model of spin-lattice coupling in Cr spinels ${ }^{414748}$. In that case the four-site cell is the tetrahedron from the which the pyrochlore lattice is built, and the role of thermal fluctuations is played by a biquadratic spin interaction.

\section{SMALL ANISOTROPY, $D=0.02$}

The phase diagram for $D=0.02$, presented in Fig. 10 , is extremely rich. It exhibits all but one of the phases catalogued in Fig. 2 - a collinear stripe phase at low values of field, giving way to either a supersolid or a spin-flop phase as magnetic field is increased; a novel 2:1:1 canted state, a collinear $m=1 / 2$ plateau state and finally, approaching saturation, a 3:1 canted phase. The key to understanding the richness of this magnetic phase diagram is to recognise that none of these phases found for $D=0$ are selected by energetic considerations alone - all of them owe their stability to thermal fluctuations. The difference in entropy between these phases and the other degenerate alternatives is, however, very small. For this reason the introduction of even a vanishingly small easy-axis anisotropy has profound consequences for the phase diagram, especially at low temperatures.

To explore how this works, we first consider the $T=0$, mean-field energies per spin of the two competing states at low magnetic field, the canted spin flop state (SPF) and the partially canted magnetic supersolid (SSD)

$$
\begin{aligned}
E_{\mathrm{SPF}} & =-J_{1}-J_{2}+J_{\perp}-\frac{h^{2}}{16\left(J_{1}+J_{2}\right)-4 D} \\
E_{\mathrm{SSD}} & =\frac{-16\left(J_{1}+J_{2}\right)\left(J_{1}+J_{2}-J_{\perp}\right)-8 D\left(J_{1}+J_{2}+J_{\perp}\right)+4 D^{2}}{16\left(J_{1}+J_{2}\right)-8 D} \\
& -\frac{h^{2}+4 D h}{16\left(J_{1}+J_{2}\right)-8 D} .
\end{aligned}
$$


Both of these state are built of the same, canted Néel sublattices, but with different relative orientations, as illustrated in Fig. 7 (c) and (d). Their energies should be compared with the (field-independent) energy of the collinear stripe state (STR)

$$
E_{\mathrm{STR}}=-J_{1}-J_{2}+J_{\perp}-D .
$$

In the absence of anisotropy (i.e. for $D=0$ ), the spin flop and supersolid states are degenerate. It is thermal fluctuations which select both the collinear stripe state in zero field, and the canted spin flop state for $h>0$. Introducing a finite anisotropy $D$ singles out the stripe phase as the ground state, and lifts the degeneracy between the canted spin flop and supersolid states. However, while thermal fluctuations favour the spin flop state, anisotropy favours the supersolid, since the spins lie closer to the easy axis. This sets up an interesting tension between energy and entropy which, for very small values of $D$, compete on an equal footing.

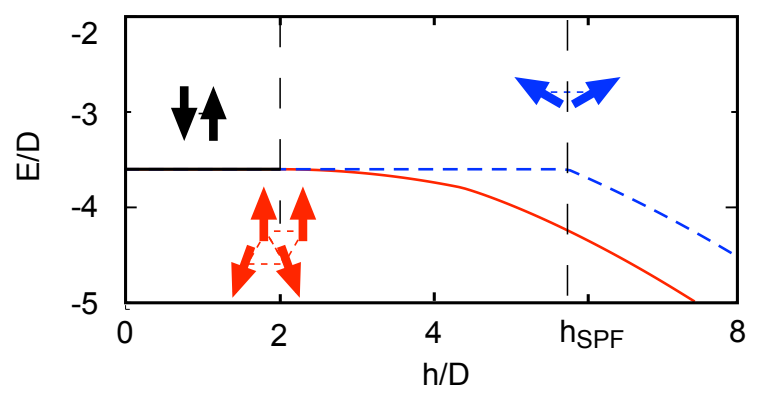

FIG. 11: (color online) Comparison of zero-temperature mean field energies for the canted spin flop (blue, dashed line) and magnetic supersolid (red solid line) states for parameters $J_{2}=0.15$ and $J_{\perp}=-0.15$ as a function of magnetic field $h$. A continuous phase transition into the supersolid state occurs at $h_{\mathrm{SSD}}=2 \mathrm{D}$, anticipating the first order spin flop transition which would otherwise have occurred at $h_{\mathrm{SPF}}$, Eq. 111.

At zero temperature phase transitions are controlled by energy alone. The first transition as a function of magnetic field is into the supersolid state, at a critical field of

$$
h_{\mathrm{SSD}}=2 D,
$$

In contrast, the field at which the spin flop state first becomes energetically favourable is

$$
h_{\mathrm{SPF}}=4 \sqrt{D\left(J_{1}+J_{2}-D / 4\right)} .
$$

Moreover, the transition into the supersolid is continuous, whereas the (avoided) spin flop transition is first order. This hierarchy of transitions is illustrated in Fig. 11.

The seeds of this supersolid state can also be found in a spin-wave expansion around the collinear state for $D>0$. At zero field this yields a gapped dispersion with minima at both the ordering momentum vector $M$ and at the symmetry related momentum $M^{\prime}$ [Fig, $\left.4(\mathrm{~b})\right]$. This degeneracy is lifted by an applied field in favour of $M^{\prime}$, with the spin gaps evolving as

$$
\begin{aligned}
\Delta_{M} & =D+2\left(J_{1}+J_{2}\right)-\frac{1}{2} \sqrt{16\left(J_{1}+J_{2}\right)^{2}+h^{2}}, \\
\Delta_{M^{\prime}} & =D-\frac{h}{2} .
\end{aligned}
$$

The gap at $M^{\prime}$ closes at a field $h_{\mathrm{SSD}}=2 D$, while the gap at $M$ would, hypothetically, close at $h_{\mathrm{SPF}}$ [Eq. [11)]. It is therefore the closing of the gap at $M^{\prime}$ which mediates the phase transition. And, since this spin wave is a transverse mode with finite momentum, it converts the collinear stripe phase into a supersolid state with a finite (staggered) magnetization in the $S^{x}-S^{y}$ plane. The spin-wave dispersion at $h=h_{\mathrm{SSD}}$, when the gap first closes, is illustrated in Fig. 12. An exactly analogous phase transition occurs in the quantum model studied with (linear) spin-wave analysis, where it is interpreted as Bose-Einstein condensation of magnons 41 , to give a condensate which breaks the transverse $U(1)$ symmetry. Similar mode-softening transitions have been observed in other models of frustrated triangular lattices 43 .

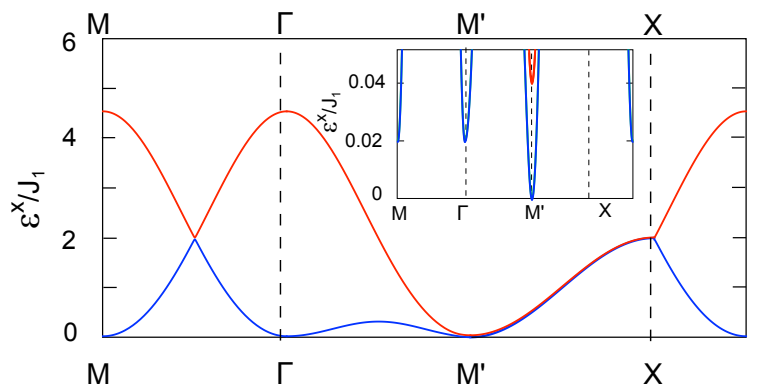

FIG. 12: (color online) Classical spin-wave dispersion $\left(S^{x}\right.$ modes only) for $J_{2}=0.15, J_{\perp}=-0.15$ and $D=0.02$, at a magnetic field $h=2 D$ associated with continuous transition from collinear stripe phase into supersolid through closing of the spin gap at $M^{\prime}$. Inset shows low-energy detail.

At finite temperature entropy again enters into the argument. Entropy favours both the collinear stripe state and the canted spin flop state over the supersolid. This has two consequences. At low temperatures, it leads to a critical field $h_{\mathrm{SSD}}(T)$ which increases with temperature. And, at higher temperatures, for sufficiently small $D$, entropy can drive a first order transition from the supersolid into the spin flop state. We can describe the first of these two effects quantitatively within a Landau theory.

In analogy with Eq. (5), we define an order parameter

$$
O_{U(1)}=\frac{1}{N} \sum_{\diamond}^{N / 4}\left|\mathbf{S}_{C}^{\perp}-\mathbf{S}_{D}^{\perp}\right|,
$$

where $C$ and $D$ label the two canted sublattices of the supersolid state, as illustrated in Fig. 2(c). The Landau expansion of the free energy can then be written as

$$
\mathcal{F}=\mathcal{F}_{0}+\frac{a}{2}\left|O_{U(1)}\right|^{2}+\frac{b}{4}\left|O_{U(1)}\right|^{4}+\ldots \quad,
$$



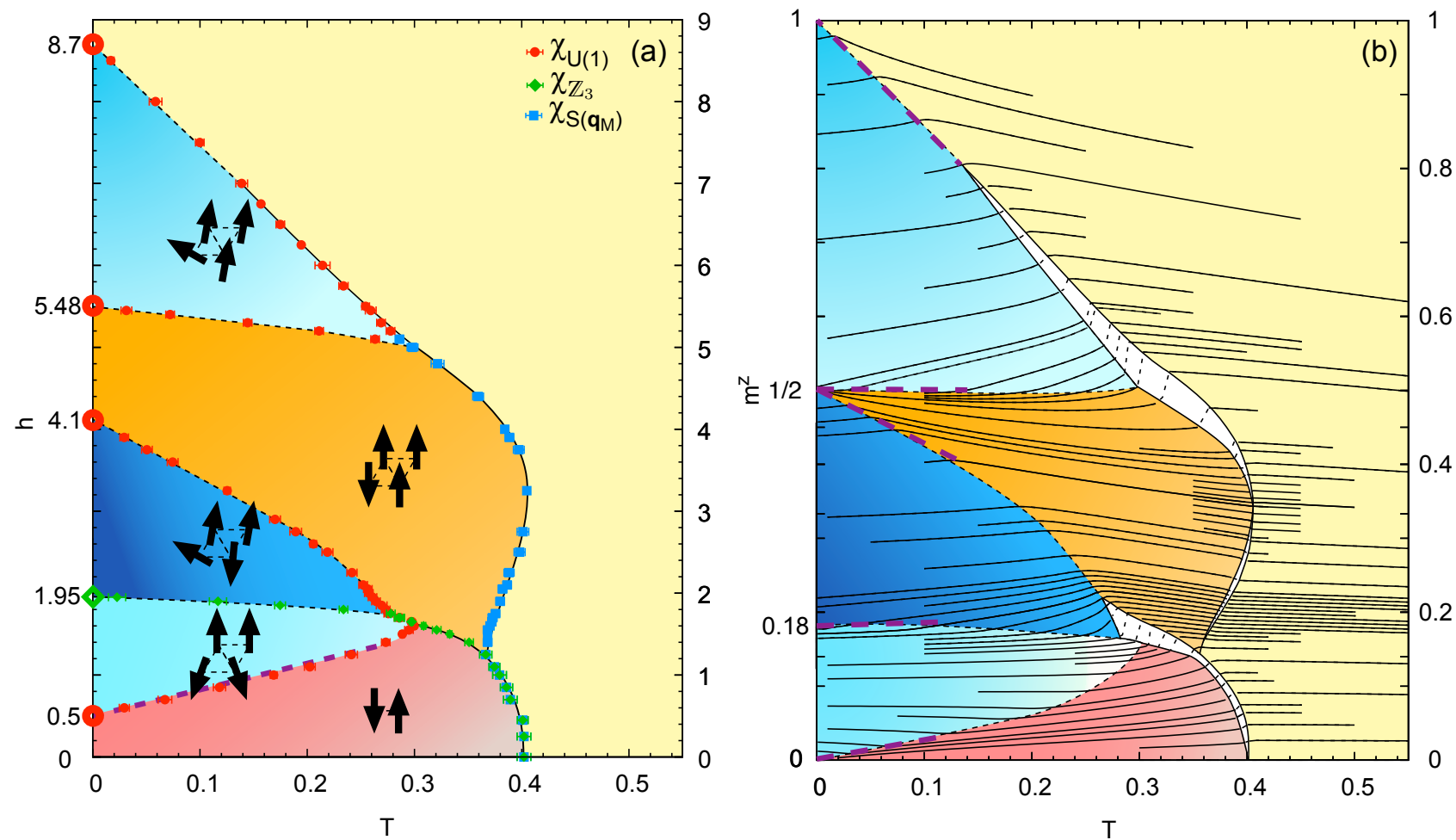

FIG. 13: (color online) Magnetic phases of a layered triangular-lattice antiferromagnet with exchange interactions $J_{1}=1, J_{2}=0.15$, $J_{\perp}=-0.15$ and easy-axis anisotropy $D=0.25$. (a) Phase diagram as a function of temperature and magnetic field. Open symbols on the h-axis show transitions obtained in mean-field theory. Phase boundaries at finite temperature are obtained from Monte Carlo simulation for a cluster of $24 \times 24 \times 8$ spins, and determined by peaks in the relevant order parameter susceptibilities. All phase transitions are first-order, except where shown with a dashed line. Thick purple dashed line is obtained through a Landau expansion for the supersolid transition. (b) Phase diagram as a function of temperature and magnetization. Solid lines running left-right show cuts at constant magnetic field $h$ taken from simulations. The coexistence regions associated with first order phase transitions are coloured white. Thick purple dashed lines show phase boundaries obtained through a low-T expansion. Easy-axis anisotropy stabilizes the new phases for a wide-range of values.

where the coefficient $a=h_{\mathrm{SSD}}(T)-h$ is calculated exactly within a spin-wave expansion about the supersolid state. Details of this are given in Appendix B, For $D=0.02$ we find a critical field of

$$
h_{\mathrm{SSD}}(T)=0.04+3.02(4) \times T .
$$

The increase of the critical field with temperature is a direct consequence of the fact that the supersolid state has a lower entropy than the collinear stripe phase. The decrease in entropy on entering the supersolid state can be traced back to a shift of spectral weight to higher energies in the spin-wave spectrum.

We can also independently estimate $h_{\mathrm{SSD}}(T)$ from the peak in the related order parameter susceptibility

$$
\chi_{O_{U(1)}}=N \frac{\left\langle O_{U(1)}^{2}\right\rangle-\left\langle O_{U(1)}\right\rangle^{2}}{T},
$$

which we calculate from Monte Carlo simulation. For $T \lesssim 0.2$ the analytic and numerical calculations are in perfect agreement, as shown by the purple dashed line in Fig. 10(a). However for $T \gtrsim 0.2$ the continuous phase transition into the supersolid phase is replaced by a firstorder transition into the entropically favoured, spin flop state.
As a direct result, there is now a finite temperature transition from the supersolid into the spin flop state for $T \approx 0.2$. As might be expected, this phase transition is strongly first order, and exhibits marked hysteresis. The character of this phase transition is most clearly seen in the coexistence region for $T \approx 0.2, m \approx 0.1$ in Fig. 10 (b). In conclusion, although the spin flop state is entirely eliminated at low temperatures by the introduction of anisotropy, its greater entropy enables it to survive at finite temperatures - for sufficiently small values of $D$. From simulations, we estimate that the spin flop phase survives at finite temperature for $D \lesssim 0.045$. The great wealth of other phases shown in Fig. 10 are also present at higher values of $D$, where simulations are easier to perform. We therefore defer the detailed analysis of these phases to the remaining sections of this paper.

\section{MODERATE ANISOTROPY, $D=0.25$}

The $D=0$ results showcase the canonical features of frustrated magnets in applied magnetic field - see e.g. Refs. 9 and 10 - a collinear plateau state is stabilized at intermediate fields by thermal fluctuations, while copla- 

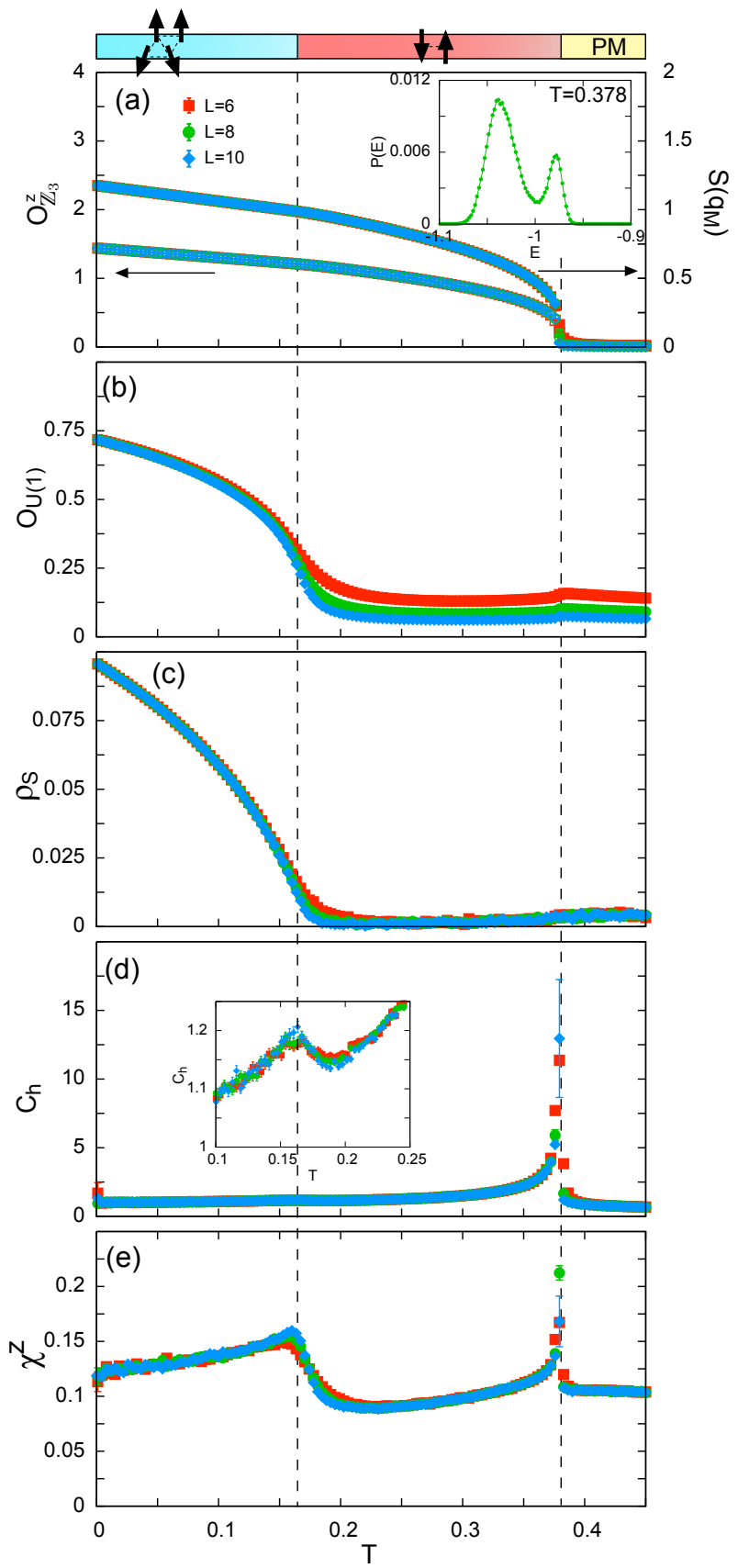

FIG. 14: (color online) Double transition from the paramagnet into the stripe collinear antiferromagnet and then into the supersolid phase for $D=0.25, h=1.0$. Translational $\mathcal{S}^{z z}\left(\mathbf{q}_{\mathrm{M}}\right)$ and rotational $\mathbb{Z}_{3}$ [Eq. 18] symmetries are broken at the same temperature $T \approx 0.37$ (a), where system transitions into the collinear phase. The first-order character of the outer transition is confirmed by the double peak in energy histograms near the critical temperature in the inset to (a). A further continuous symmetry is broken at $T \approx 0.16$ at the onset of the supersolid phase, as observed in the $U(1)$ order parameter (b) and spin stiffness (c). The different nature of these transitions is clear in heat capacity (d) and magnetic susceptibility (e).

nar canted phases are found at both lower and higher fields. However, in this model even a very small easyanisotropy leads to strikingly different results, as demon- strated for $D=0.02$. We now analyse thoroughly the phases driven by anisotropy for a representative, moderately small value of anisotropy $D=0.25$. Our results are summarized in the phase diagrams shown in Fig. 13. For this value of $D$, the spin flop phase is entirely suppressed, and the low temperature physics is dictated by considerations of energy, rather than entropy. As a function of increasing magnetic field, we find a collinear stripe state, the supersolid phase described above, an exotic $2: 1: 1$ canted state, a collinear $m=1 / 2$ plateau and finally a 3:1 canted state which interpolates to saturation. This large set of phases gives rise to a correspondingly large number of phase transitions, which we analyze below.

We begin by considering the finite-temperature phase transition from the paramagnet into the collinear stripe state at low values of magnetic field, and the subsequent low temperature transition from the stripe phase into the supersolid. Both of these transition may have been observed in $\mathrm{AgNiO}_{2}$ [Ref. 39]. In Fig. 14 we show a selection of simulation results for $h=1.0$, and a range of temperatures spanning all three phases. The transition from paramagnet to collinear stripe phase at $T=0.379(4)$ is evident in sharp peaks in both the heat capacity [Fig. 14(d)] and the magnetic susceptibility [Fig. 14(e)]. This transition is first order, as evidenced by a bimodal energy histogram [inset to Fig. 14(a)]. The collinear stripe phase breaks both the translational and the rotational symmetry of the lattice. Both of these symmetries are broken at the same temperature, as demonstrated in Fig. 14(a) by the temperature dependence of the spin structure factor $\mathcal{S}^{z z}\left(\mathbf{q}_{\mathrm{M}}\right)$ and a suitably modified order parameter for lattice rotations

$$
O_{\mathbb{Z}_{3}}^{z}=\left\langle\sqrt{\left|\psi_{1}^{z}\right|^{2}+\left|\psi_{2}^{z}\right|^{2}}\right\rangle
$$

where

$$
\begin{aligned}
& \psi_{1}^{z}=\frac{1}{\sqrt{6} N} \sum_{i} 2 S_{i}^{z} \cdot S_{i+\delta_{1}}^{z}-S_{i}^{z} \cdot S_{i+\delta_{2}}^{z}-S_{i}^{z} \cdot S_{i-\delta_{1}-\delta_{2}}^{z} \\
& \psi_{2}^{z}=-\frac{1}{\sqrt{2} N} \sum_{i} S_{i}^{z} \cdot S_{i+\delta_{2}}^{z}-S_{i}^{z} \cdot S_{i-\delta_{1}-\delta_{2}}^{z} \cdot
\end{aligned}
$$

The first order behaviour of the phase transition seen here chimes with the known first order phase transition from the paramagnet into the collinear stripe phase of $\mathrm{AgNiO}_{2}$ [Ref. 40].

The second, internal, phase transition at $T=0.171(4)$ from collinear stripe into the supersolid state is continuous, exhibiting only shows weak anomalies in specific heat [Fig. 14(d)] and magnetic susceptibility [Fig. 14(e)]. The new broken symmetry - a staggered in-plane magnetization - is heralded by the smooth rise of both the $U(1)$ order parameter $O_{U(1)}$ [Fig. 14(b)] and the spin stiffness [Fig. 14(c)]. In this context, the susceptibility associated with the $U(1)$ order parameter can be analysed with a finite-size scaling ansatz

$$
\chi_{O_{U(1)}}=L^{\gamma / \nu} \tilde{\chi}\left(L^{1 / \nu} t\right)
$$



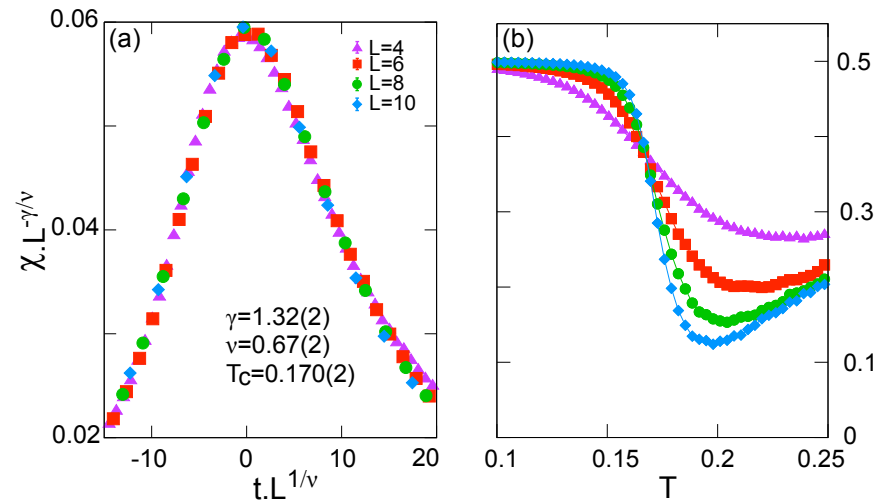

FIG. 15: (color online) Continuous behaviour of the collinearsupersolid transition for $D=0.25, h=1.0$ at $T \approx 0.17$. (a) Data collapse for the supersolid $U(1)$ order parameter susceptibility using 3D XY universality class critical exponents. (b) The Binder cumulant for the same order parameter calculated for different lattice sizes all cross at a single temperature $T=0.168(1)$.

where $t$ is the reduced temperature $t=\left(T-T_{c}\right) / T_{c}$. The critical exponents associated with the order parameter susceptibility $(\gamma)$, and correlation length $(\nu)$ are extracted by fitting this expression to simulation results for different lattice sizes [Fig. 15. The exponents obtained, $\nu=1.32(2)$ and $\gamma=0.67(2)$, are in excellent agreement with the 3D XY universality class.

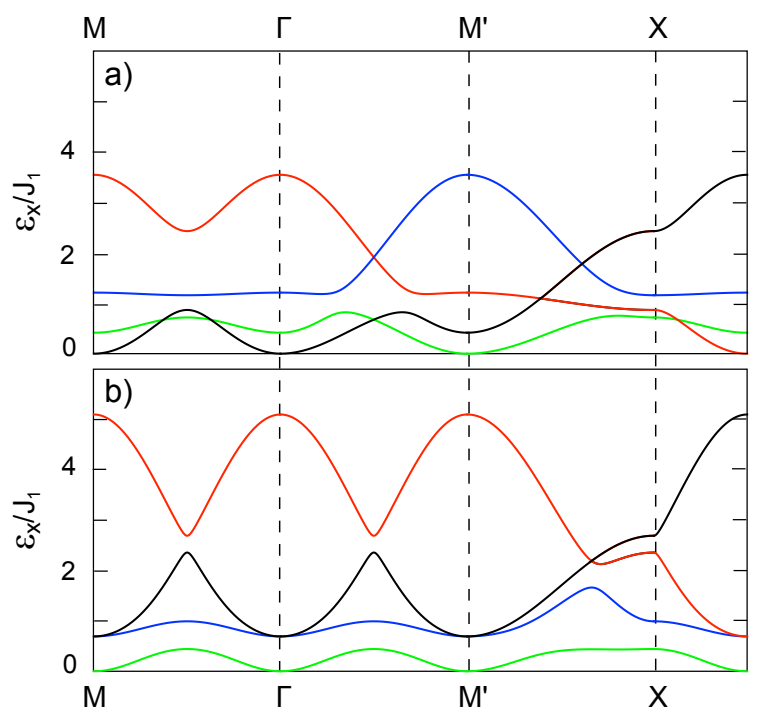

FIG. 16: (color online) Classical spin-wave dispersion $\left(S^{x}\right.$ modes only) at $D=0.25$ for values of magnetic field associated with continuous transitions at high field. (a) Closing of the spin gap at $M$ for $h=1.95$, associated with transition from the supersolid phase into the 2:1:1 phase. (b) Closing of the spin gap at $\Gamma$ for $h=5.48$, associated with transition from the $m=1 / 2$ plateau into the $3: 1$ canted phase. The points $M, M^{\prime}$ and $\Gamma$ are defined in Fig. 4 (b).

In previously studied models with $X X Z$ anisotropy, supersolid phases were found as intermediate phases interpolating between Néel antiferromagnetic and spinflop phases 27/37. However, in the present model, a different phase emerges above the supersolid. This is a
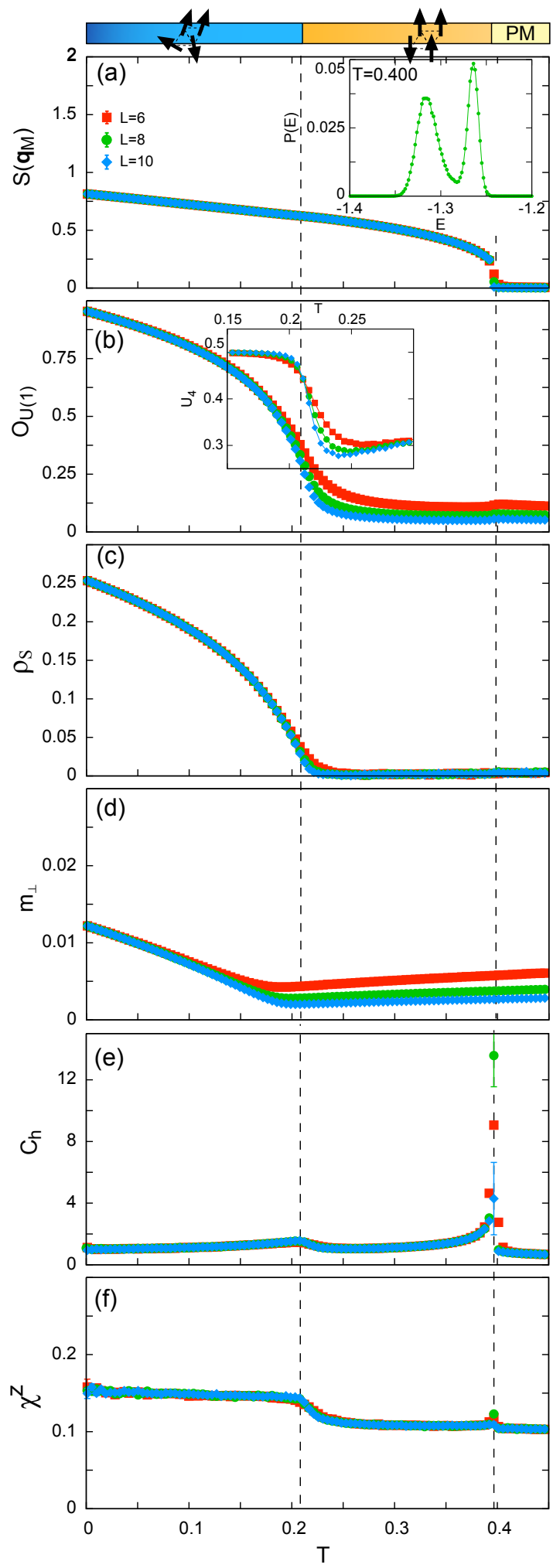

FIG. 17: (color online) Double phase transition at $D=0.25, h=2.5$, from the paramagnet into $m=1 / 2$ plateau and then into 2:1:1 canted state at lower temperature. (a) Translational symmetry measured by $\mathcal{S}^{z z}\left(\mathbf{q}_{\mathrm{M}}\right)$ is broken at $T \approx 0.4$ in the first-order transition - cf. bimodal energy distribution (inset) - into the plateau. (b) $U(1)$ continuous symmetry and (c) spin stiffness are broken in the continuous transition - cf. crossing of $U(1)$ order parameter Binder cumulants in inset to (b) - from the plateau into the canted phase at $T \approx 0.21$. (d) The 2:1:1 canted state is also characterised by in-plane magnetisation $m_{\perp}$ (d). The different nature of these transitions is resolved in heat capacity (e) and magnetic susceptibility (f). 
2:1:1 canted phase with a four-spin unit cell where two spins are parallel to each other and have positive $S^{z}$, while a third is orientated in the negative $S^{z}$ direction, and the remaining spin rotates between those positions [Fig. 2(e)]. At $T=0$ this transition is continuous, and is observed in both mean-field and spin-wave calculations, which reveal a soft mode at momentum $M$ [Fig. [16(a)]. With further increase in magnetic field, the 2:1:1 canted phase evolves smoothly into to the collinear $m=1 / 2$ plateau.

Since the 2:1:1 canted phase breaks both translational and in-plane symmetries it can also be labelled as a supersolid. Furthermore, it possesses a (very small) moment

$$
m_{\perp}=\frac{1}{N}\left\langle\left|\sum_{i} \mathbf{S}_{i}^{\perp}\right|\right\rangle .
$$

in the $S^{x}-S^{y}$ plane. This behaviour distinguishes it from the lower-field supersolid state, but is also seen in the easy-axis nearest-neighbour triangular lattice, where it was attributed to the non-trivial degeneracy of the $T=0$ ground state ${ }^{21}$. In addition to $m_{\perp}$, this phase is characterised by finite values of the staggered in plane magnetization, spin stiffness $\rho_{S}$ and structure factor $\mathcal{S}^{z z}\left(\mathbf{q}_{\mathbf{M}}\right)$.

In Fig. 17 we present simulation results for $h=2.5$, for a range of temperatures spanning the paramagnetic, $m=1 / 2$ plateau and 2:1:1 canted phases. The phase transition from the paramagnet to the $m=1 / 2$ plateau is marked by an abrupt rise in $\mathcal{S}^{z z}\left(\mathbf{q}_{\mathrm{M}}\right)$ at $T=0.397(4)$. The transition is first order, as evidenced by a bimodal energy histogram [inset to Fig. 17(a)], and accompanied by a sharp feature in the heat capacity [Fig. 17(e)]. The phase transition from the $m=1 / 2$ plateau to the 2:1:1 canted state is continuous, with the Binder cumulants for the associated $U(1)$ order parameter crossing at $T=0.211$ (1) in inset to Fig. 17(b).

This continuous phase transition is mediated by a soft spin wave mode within the collinear $m=1 / 2$ plateau, just as the transition into the supersolid is mediated by a soft spin-wave mode within the collinear stripe phase. In this case the relevant spin-wave gap occurs at the zone centre, and closes with decreasing magnetic field, at a $T=0$ critical field of

$$
h_{c}=4\left(J_{1}+J_{2}\right)-2 D .
$$

Easy-axis anisotropy stabilizes the plateau at $T=0$ for a finite range of field, and its collinearity ensures it is entropically favoured against the 2:1:1 canted state at finite temperature. The critical field therefore slopes downward with increasing temperature - the opposite of what is seen in the transition from the collinear stripe phase into the supersolid [Fig. 13 .

The transition from the collinear $m=1 / 2$ plateau into the $3: 1$ canted state at high field also occurs through the condensation of a zone-centre spin wave mode [Fig. 16(b)], this time at a $T=0$ critical field of

$$
h_{c}=2\left(J_{1}+J_{2}\right)+2 \sqrt{D^{2}+4 D\left(J_{1}+J_{2}\right)+\left(J_{1}+J_{2}\right)^{2}} .
$$
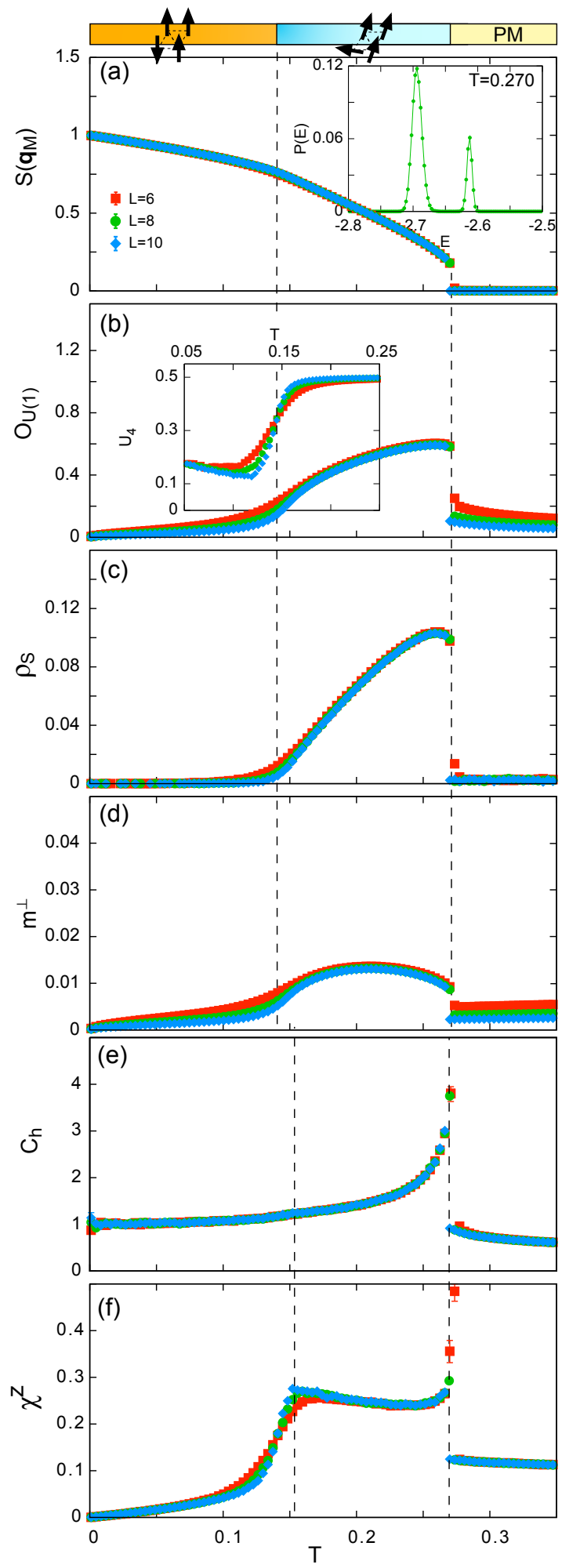

FIG. 18: (color online) Double phase transition at $D=0.25, h=5.3$ from the paramagnet to $3: 1$ canted state and then into $m=1 / 2$ plateau. The $3: 1$ canted state is heralded by its discrete $\mathcal{S}^{z z}\left(\mathbf{q}_{\mathrm{M}}\right)$ (a) and continuous $\mathrm{U}(1)$ order parameters (b), and also by finite spin stiffness (c). This state also has a finite in-plane magnetisation $m_{\perp}$ (d). The upper transition first-order character is clear in the double-peaked energy energy distribution close to the critical temperature in inset to (a), while the continuous character of the lower transition is shown by the Binder cumulant for the $U(1)$ order parameter, in inset to (b). The different nature of these transitions is resolved in heat capacity (e) and magnetic susceptibility (f). 


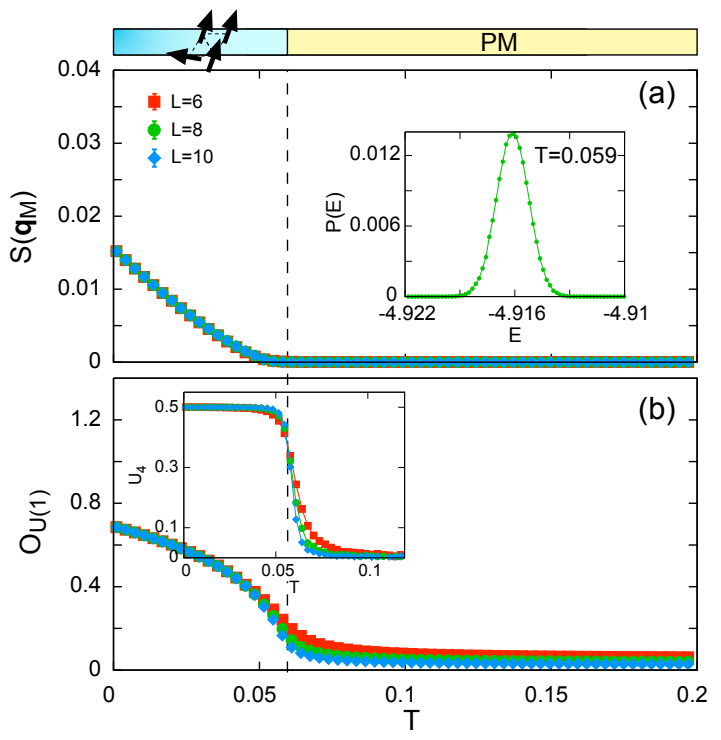

FIG. 19: (color online) Temperature dependence of order parameters associated with 3:1 canted phase close to saturation. The continuous nature of the phase transition is confirmed by the singlevalued energy histogram close to the critical temperature in inset to (a), and crossing of Binder cumulants associated with $U(1)$ order parameter in inset to (b).

This critical field is only weakly dependent on temperature and, perhaps surprisingly, slopes downwards [Fig. 13. As noted in Section III] the 3:1 canted phase breaks both discrete translational and continuous spin rotational symmetries [cf. Fig. 18]. It is therefore a third supersolid, in the sense of Matsuda and Tstuneto or Liu and Fisher. However, in contrast to the $D=0$ case, for finite anisotropy the $3: 1$ canted phase possesses a finite value of in-plane magnetisation $m_{\perp}$.

The $3: 1$ canted state is the only phase with supersolid character which is directly connected to the paramagnetic region. This phase transition is clearly firstorder at low field, e.g. $h=5.3$, from simulation results [Fig. 18]. Nevertheless a continuous phase transition is permitted by symmetry, and the transition at high fields

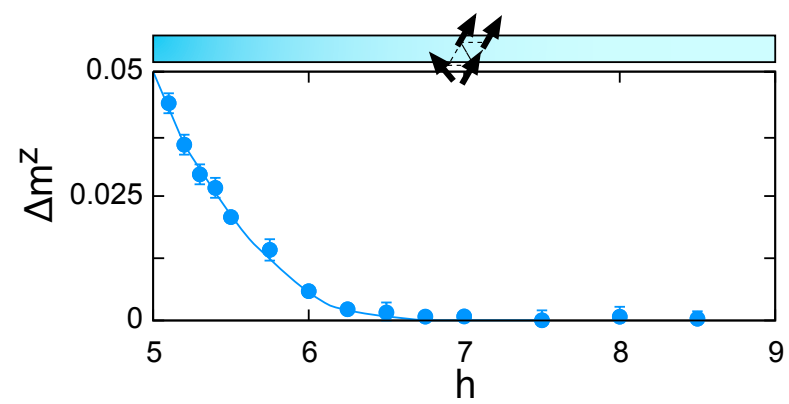

FIG. 20: (color online) Vanishing discontinuities in magnetisation for $D=0.25$, extracted from temperature cuts in Fig. 13 indicate a crossover from first to second-order phase transition at the paramagnet - 3:1 canted state transition, where the critical endpoint is found at $T \approx 0.14, h \approx 6.5$. Line is a guide to the eye. is indeed continuous [Fig. 19. At $T=0$ it is easy to see that this continuous transition corresponds to the opening of a spin-wave gap at the 4-sublattice ordering vector(s) $\left\{\mathbf{q}_{\mathrm{M}}\right\}$, within the saturated state. For $T=0$, this occurs at

$$
h_{\mathrm{SAT}}=8\left(J_{1}+J_{2}\right)-2 D .
$$

and the critical field slopes sharply downwards, as required by the higher entropy of the paramagnetic phase. We conclude that fluctuations drive this continuous phase transition first order for $T \gtrsim 0.12(h \lesssim 6.5)$ - the point at which a finite jump in the magnetization $\Delta m^{z}$ is first observed when going from the $3: 1$ canted phase to paramagnet [Fig. 20].

Up to this point we have chiefly concentrated on symmetry breaking at low temperatures, where simulation results can be reliably compared with mean-field theory and spin-wave calculations. We now turn to the more delicate question of how the full symmetry of the system is recovered with increasing temperature, starting from the low-field supersolid phase. In the original finite-temperature scenario of Liu and Fisher, the supersolid phase extends all the way to the paramagnet,
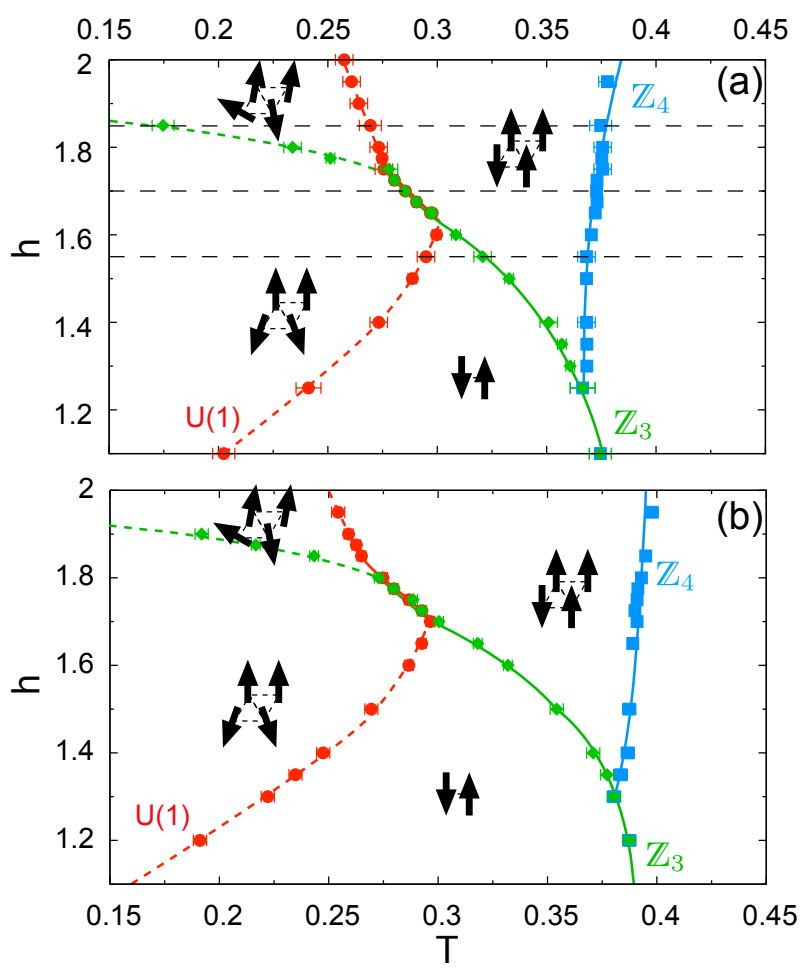

FIG. 21: (color online) Region where four phases compete at intermediate field for $D=0.25$ (a) and $D=0.325$ (b) for system size $L=8$. Phase transitions are labelled according to the symmetries broken. Three distinct critical points are identified in a narrow field range, however these never merge into a single tetracritical point. This structure is insensitive to changes in the easy-axis anisotropy as long as the $T=0$ mean field analysis yields the same phases. All phase transitions are first-order, except where shown with a dashed line. Dashed horizontal lines in (a) show cuts at fixed field studied in Fig. 22 

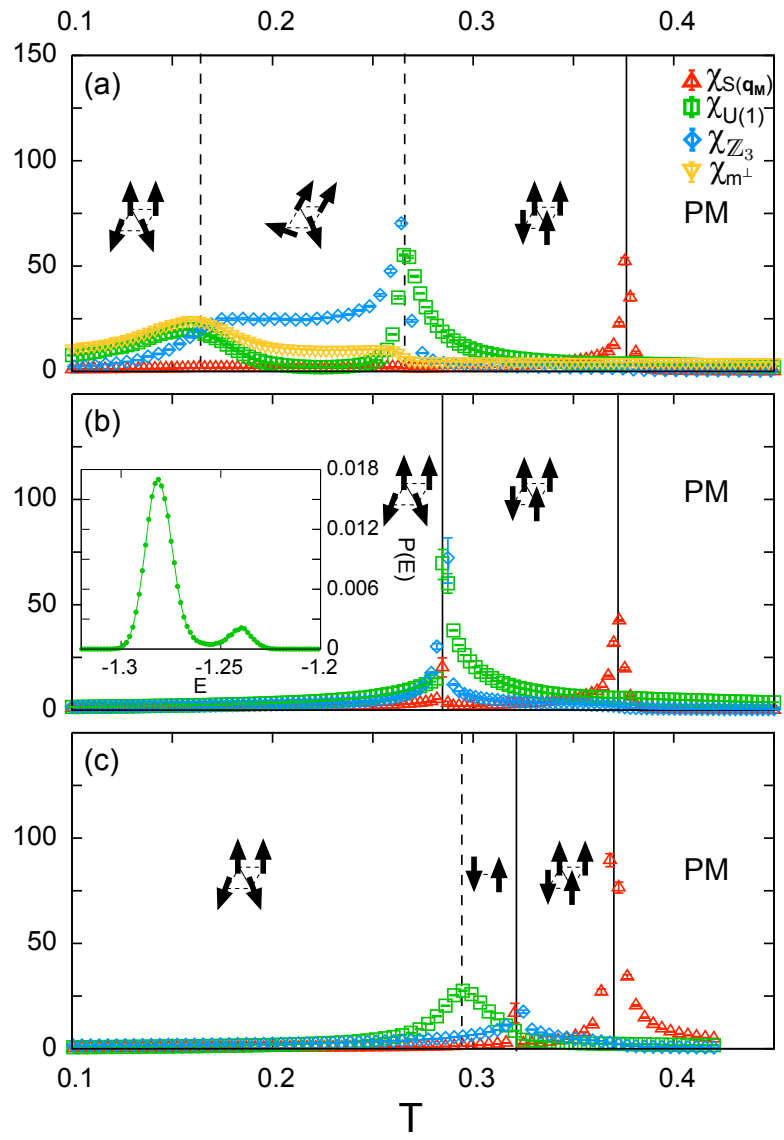

FIG. 22: (color online) Successive phase transitions at $D=0.25$ for system size $L=8$ as a function of field as resolved in the susceptibilities associated with breakdown of translational symmetry $\mathcal{S}^{z z}\left(\mathbf{q}_{\mathrm{M}}\right)$, spin-rotation symmetry $U(1)$, lattice rotation symmetry $\mathbb{Z}_{3}$ and in-plane magnetisation $m_{\perp}$. (a) At high field, $h=1.85$, the $m=1 / 2$ plateau transforms into the $2: 1: 1$ canted state, followed by the supersolid phase. (b) For intermediate field, $h=1.70$, there is a direct first-order phase transition from the $m=1 / 2$ plateau into the supersolid state, as evidenced by the coincidence the susceptibility peaks at $T \approx 0.28$. (c) At lower field, $h=1.55$, the system transitions from paramagnet into $m=1 / 2$ plateau, then into the stripe phase and finally into into supersolid state. Phase transitions are first order except where indicated with dashed line.

where it terminates in a tetracritical point ${ }^{27}$. Exactly at this tetracritical point four phases (collinear antiferromagnet, supersolid, spin-flop and paramagnet) meet, and all phase transitions between them are continuous. The behaviour of the present model at high temperatures is markedly different. From the phase diagrams shown in Fig. 13 it is clear that the collinear phases completely engulf the non-collinear ones at high temperature due to their higher entropy, so the supersolid phase never touches the paramagnet. However, for intermediate values of field four phases - the collinear stripe phase, supersolid, 2:1:1 canted phase, and the collinear $m=1 / 2$ plateau - are found in very close proximity to one another. It is therefore worth asking whether any other multicritical points arise in this model.

In Fig. 21(a), we present a detailed study of the in- termediate field region, $1.2<h<2$. Associated field cuts for $D=0.25$ are shown in Fig. 22 . The $\mathbb{Z}_{3}$ transition between the two collinear states is clearly first order, while the transition between the supersolid and 2:1:1 canted phases is continuous. The phase transition between the collinear stripe state and the supersolid terminates on the $\mathbb{Z}_{3}$ line. The transition between the 2:1:1 canted state and the collinear $m=1 / 2$ plateau is continuous at high fields, but becomes first order shortly before terminating on the $\mathbb{Z}_{3}$ line for $h=1.76(1)$ [Fig. 23. For $h=1.70$ [Fig. 22(b)], there is a clear first order transition between the $m=1 / 2$ plateau and supersolid, where all observed susceptibility peaks merge. Given the difficulty in simulating this parameter region with many neighbouring phases, the possibility of a vanishingly narrow "strip" of 2:1:1 canted phase extending between the supersolid and plateau phases is hard to rule out definitely. However, we find no evidence of finite $m_{\perp}$, characteristic of the 2:1:1 state, in this range of temperature and field.

From this analysis we conclude that the majority of these phase transitions remain first order where the four phases converge, and that no more than two phases meet at a single point via a continuous phase transition. Even if the 2:1:1 canted phase were to stretch to lower fields, the first-order nature of the phase transitions between the $m=1 / 2$ plateau and 2:1:1 canted phases, and $m=1 / 2$ plateau and collinear stripe phases would preclude a tetracritical point - at most we would have a "bicritical endpoint", where two continuous phase transitions meet two first-order ones. This behaviour persists throughout the anisotropy range where these four phases are present, as illustrated in Fig. 21.(b) for $D=0.325$.

In the same spirit, it is worth re-examining the finite temperature transition between the paramagnet and collinear $m=1 / 2$ plateau. While generically first-order, there exist two points in the phase diagram, $h \approx 1.25$ and $h \approx 3.2$, where the jump in magnetisation between the two phases vanishes, indicating the possibility of a continu-

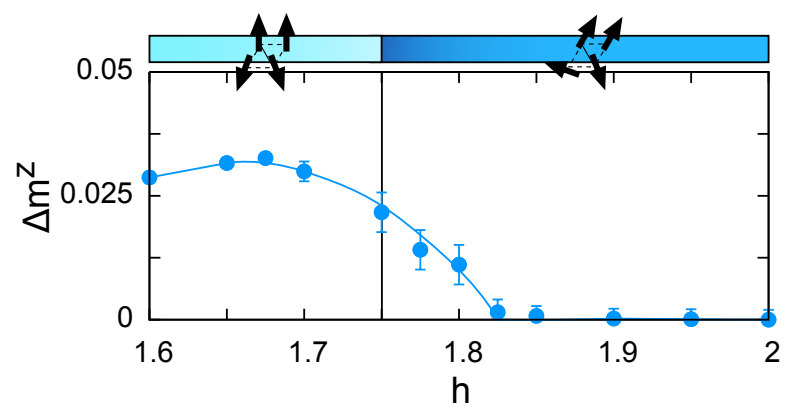

FIG. 23: (color online) Discontinuities in magnetisation $\Delta m^{z}$ on entering the $m=1 / 2$ plateau from the supersolid and 2:1:1 canted phases for $D=0.25$ and intermediate field, extracted from temperature cuts in Fig. 13 The collapse in $\Delta m^{z}$ for $h=1.81(2)$ indicates that the transition from the $2: 1: 1$ canted phase to the $m=1 / 2$ plateau becomes first order before merging with the transition from the supersolid to the $m=1 / 2$ plateau. This precludes a multicritical point. The line is a guide to the eye. 

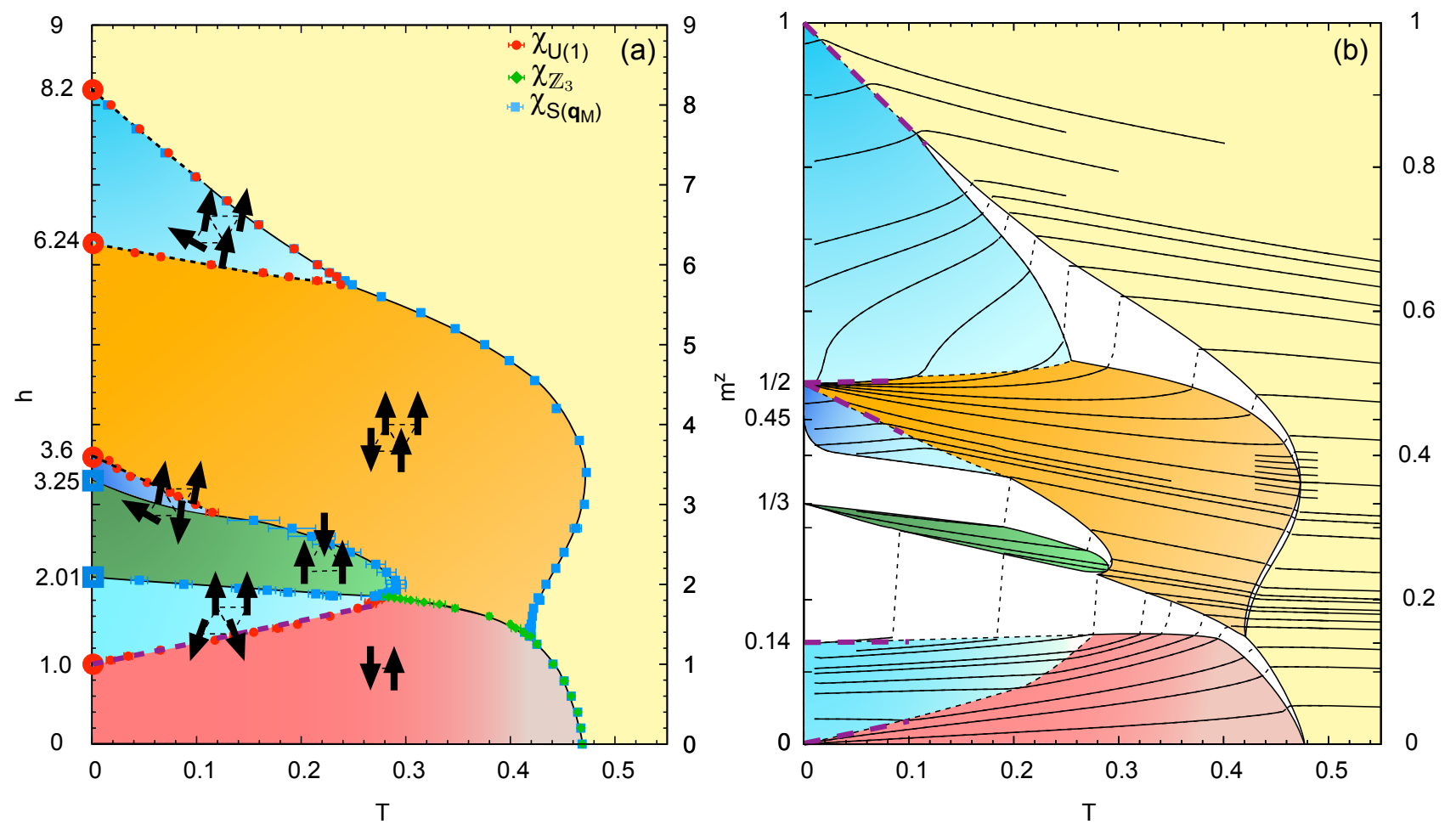

FIG. 24: (color online) Magnetic phases of a layered triangular-lattice antiferromagnet with $J_{1}=1, J_{2}=0.15, \quad J_{\perp}=-0.15$ and easy-axis anisotropy $D=0.5$. (a) Phase diagram as a function of temperature and magnetic field. Open symbols on the h-axis show transitions obtained in mean-field theory. Phase boundaries at finite temperature are obtained from Monte Carlo simulation for a cluster of $24 \times 24 \times 8$ spins, and determined by peaks in the relevant order parameter susceptibilities. All phase transitions are first-order, except where shown with a dashed line. Thick purple dashed line is obtained through a Landau expansion for the supersolid transition. (b) Phase diagram as a function of temperature and magnetization. Solid lines running left-right show cuts at constant magnetic field $h$ taken from simulations. The coexistence regions associated with first order phase transitions are coloured white. Thick purple dashed lines show phase boundaries obtained through a low-T expansion. Increasing anisotropy gives rise to an $m=1 / 3$ plateau with a different unit-cell from the other phases.

ous behaviour [Fig. 13(b)]. These two points are a robust feature of the $m=1 / 2$ plateau for all values of $D$ investigated. One possible scenario for this behaviour would be the emergence of a higher symmetry at these special points. Sadly, however, this attractive scenario does not survive a closer examination of the data.

The first point at which $\Delta m^{z}=0$, at $h \approx 1.25$, appears to be a critical end point, where the first order transition between the collinear $m=1 / 2$ plateau and the paramagnet terminates on the first order transition into the collinear stripe phase. This critical end point is distinguished by a unimodal energy histogram. The second point at which $\Delta m^{z}=0$ occurs for $h \approx 3.20$, and can be understood in terms of the two different spin wave excitations which connect the collinear $m=1 / 2$ plateau with the 2:1:1 canted at lower field, and with the $3: 1$ canted state at higher field. The spin wave-excitation associated with the 2:1:1 canted state lowers the magnetization of the system, while the excitation associated with the 3:1 canted state raises the magnetization of the system.

These spin waves will also determine the sign of the magnetization jump at the finite temperature transition from the collinear $m=1 / 2$ plateau into the paramagnet. It follows that there will exist a value of magnetic field at which this jump $\Delta m^{z}$ changes sign, without any dynamically generated symmetry entering into the problem. A close examination of energy histograms and the spin structure factor $\mathcal{S}(\mathbf{q})$ at the transition bears out this interpretation.

\section{INTERMEDIATE ANISOTROPY, $D=0.5$}

In order to investigate the robustness of the $D=0.25$ results we increase the anisotropy, focusing on a representative value $D=0.5$. As a consequence a new collinear phase emerges at intermediate fields. This is the familiar three-sublattice state with two spins "up" and one "down" [Fig. 2(d)], i.e. a collinear one-third magnetisation plateau with ordering vectors at the corners of the Brillouin Zone [Fig 4(d)]. In this case the system sacrifices exchange energy associated with the secondneighbour interaction to increase its collinearity.

This $m=1 / 3$ plateau first appears within the 2:1:1 canted phase for $D>0.33$, splitting the canted phase in two. Increasing anisotropy rapidly suppresses the "lower" $2: 1: 1$ canted state so that the $m=1 / 3$ plateau becomes the third phase under field, above the supersolid. 
While in the purely nearest-neighbour case this plateau emerges continuously connected to canted phases 21 , in this situation it is completely unrelated by symmetry to the surrounding phases. Thus this $m=1 / 3$ can be thought of as an "accident" within the natural progression of four-sublattice phases.

The low-field results at $D=0.5$ have been analysed elsewhere ${ }^{41}$ and essentially reproduce the $D=0.25$ case. At $T=0$ the first-order phase transition from the supersolid into the $m=1 / 3$ plateau occurs for a mean-field field of

$$
\begin{aligned}
h= & \left(D+8\left(J_{1}+J_{2}\right)\right) / 3 \\
& -4 / 3 \sqrt{2\left(8 J_{2}-D-J_{1}\right)\left(D-2\left(J_{1}+J_{2}\right)\right)} .
\end{aligned}
$$

To study this plateau we use the $S^{z}-S^{z}$ structure factor associated with the three-sublattice ordering vectors $\left\{\mathbf{q}_{\mathrm{k}}\right\}$, cf. Fig. 4 .

$$
\mathcal{S}^{z z}\left(\mathbf{q}_{\mathrm{K}}\right)=\left\langle\sum_{\left\{q_{K}\right\}}\left|\frac{1}{N} \sum_{i} S_{i}^{z} \mathrm{e}^{-i \mathbf{q}_{\mathrm{K}} \cdot \mathbf{r}_{i}}\right|^{2}\right\rangle .
$$

At finite temperature the $m=1 / 3$ plateau is connected through first-order transitions to the $m=1 / 2$ plateau [Fig. 25], supersolid and 2:1:1 state phases. These low-temperature, strongly first-order transitions between phases with different symmetries are very difficult to simulate, even employing the combined parallel tempering and over-relaxation procedure.

For this value of anisotropy the 2:1:1 canted state survives above the $m=1 / 3$ plateau for a $T=0$ field range of $3.25<h<3.60$. While at high field the tendency of the $m=1 / 2$ plateau to cant through the closing of a spingap wins, at lower field the $m=1 / 3$ plateau is favoured entropically against the canted state. Because these two transitions lie very close to each other at low temperature, it is much more difficult to find them with the same accuracy as used in the rest of the phase diagram.

The nature of the transitions into the supersolid phases is unaffected by the increase of anisotropy. However the magnetisation jumps [Fig. 24(b)] have become more dramatic. Hence the points with $\delta m^{z}=0$ connected to the $m=1 / 2$ plateau, at $h \approx 1.4$ and $h \approx 3.6$, become more visible.

The endpoint of the inner plateau-plateau transition is very close to the critical point of the supersolid transition at $h \approx 2, T \approx 0.3$, in a shape curiously reminiscent of Fig. 21. The hysteresis associated with these strongly first-order transitions, especially the plateau-plateau one, makes a more in-depth analysis of this region difficult to perform. Nevertheless, as we shall see below, this feature is not robust for other values of anisotropy.

Another possibly interesting feature is that at an higher field of $h \approx 1.90$ the plateau-plateau transition also supports a point for which the magnetisation discontinuity apparently vanishes [Fig. 25]. As in the previous case, we find no evidence of the emergence of higher symmetries or other exotic behaviour at this point.

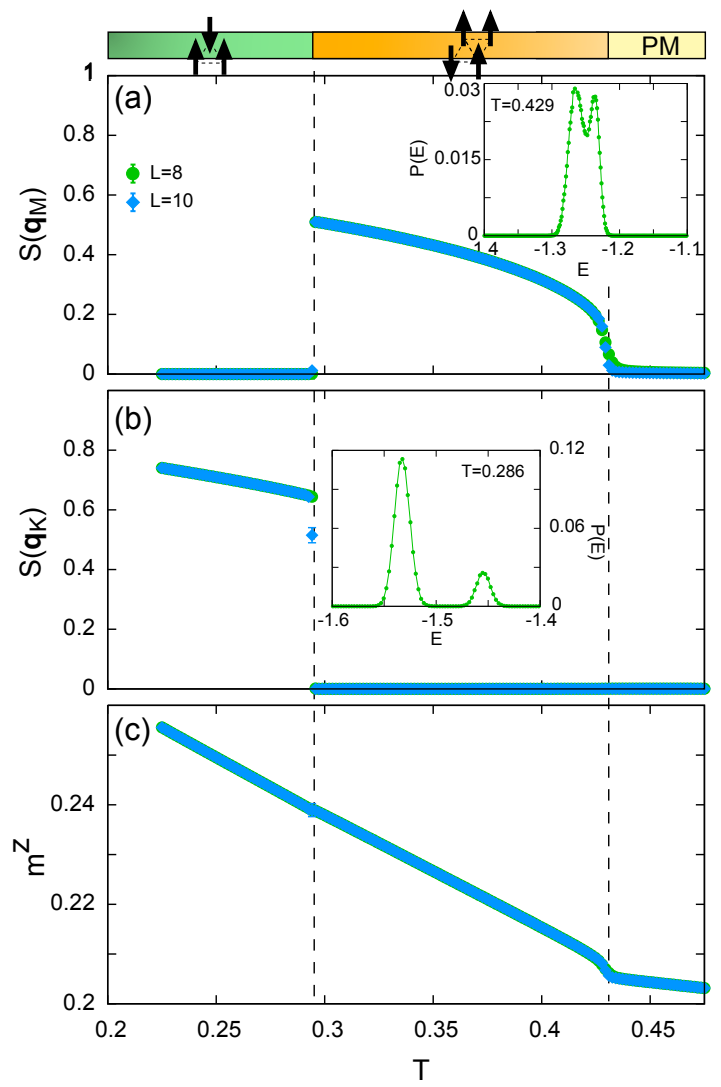

FIG. 25: (color online) Double phase transition from the paramagnet to $m=1 / 2$-plateau and then into the $m=1 / 3$-plateau at $D=0.5, h=1.89$. Temperature dependence of the structure factor measured at momenta corresponding to four-sublattice ordering $\mathcal{S}^{z z}\left(\mathbf{q}_{\mathrm{M}}\right)$ (a), and (b) momenta corresponding to threesublattice ordering $\mathcal{S}^{z z}\left(\mathbf{q}_{\mathrm{K}}\right)$ (b). Both paramagnet- $m=1 / 2$-plateau and plateau-plateau phase transitions are first-order, as seen in the energy histograms in insets to (a) and (b), however the magnetisation (c) does not exhibits the characteristic jump across the inner transition.

\section{INTERMEDIATE TO STRONG ANISOTROPY,$D=\mathbf{0 . 6 7 5}$}

By further increasing the anisotropy strength we wish to verify the stability of the $D=0.5$ conclusions, especially in the region surrounding the $m=1 / 3$ plateau. We expect the collinear phases to gain ground and eventually suppress the supersolid states. Therefore we choose to study $D=0.675$ as a representative value. We note that the set of parameters with this value of $D$ gives the bet fit to the magnetic excitations in $\mathrm{AgNiO}_{2}{ }^{42}$.

The general shape of the phase diagram [Fig. 26] is similar to the previous ones. The transition fields have become less dependent on temperature and the phases themselves are more widely separated in the magnetisation-temperature phase diagram, a reflection of the stronger anisotropy.

The main difference when compared to lower anisotropy is the complete suppression of the $2: 1: 1$ canted phase. This phase ceases to exist at $T=0$ for $D>0.625$ 

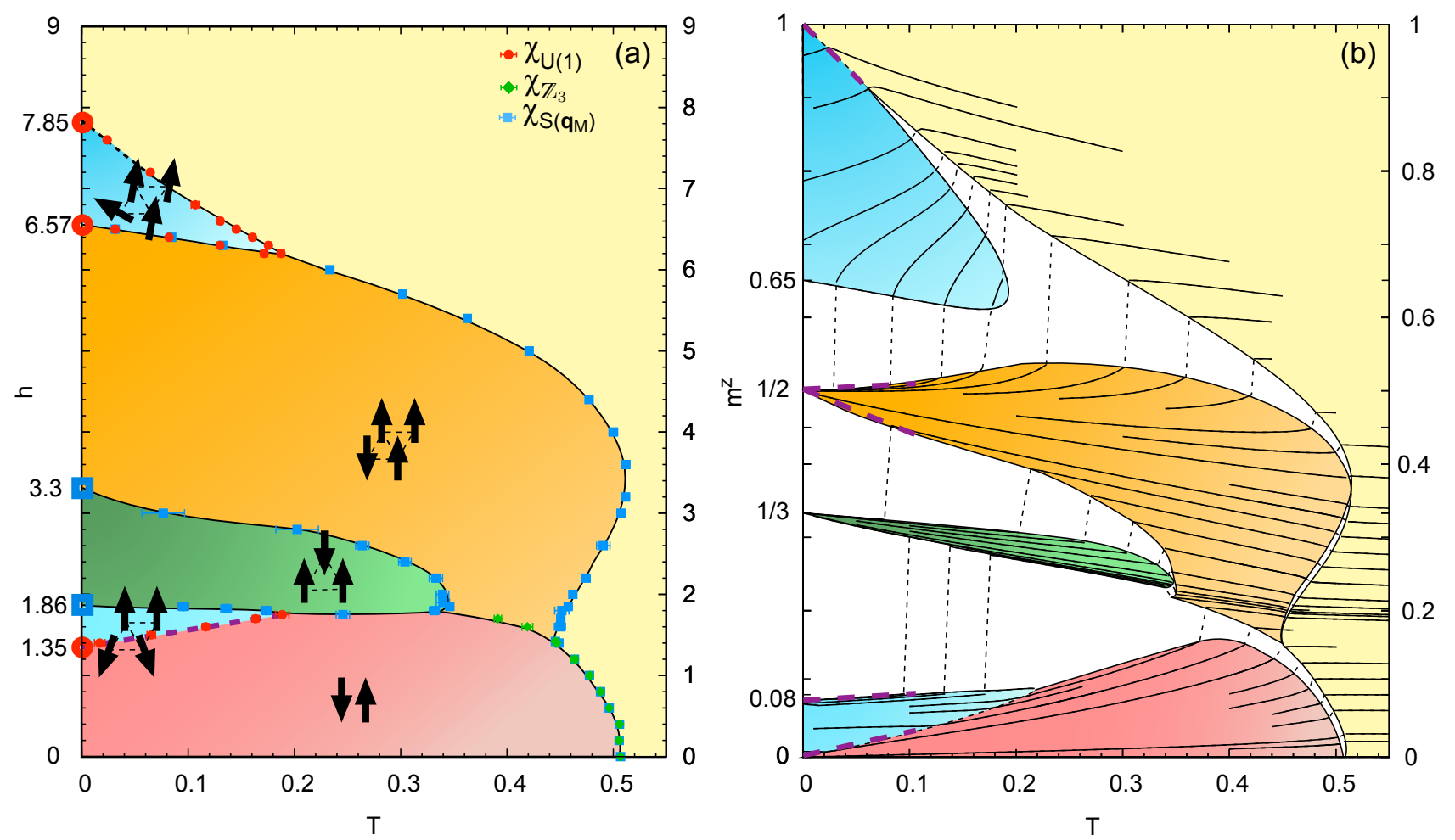

FIG. 26: (color online) Magnetic phases of a layered triangular-lattice antiferromagnet with $J_{1}=1, J_{2}=0.15, J_{\perp}=-0.15$ and easy-axis anisotropy $D=0.675$. (a) Phase diagram as a function of temperature and magnetic field. Open symbols on the h-axis show transitions obtained in mean-field theory. Phase boundaries at finite temperature are obtained from Monte Carlo simulation for a cluster of $24 \times 24 \times 8$ spins, and determined by peaks in the relevant order parameter susceptibilities. All phase transitions are first-order, except where shown with a dashed line. Thick purple dashed line is obtained through a Landau expansion for the supersolid transition. (b) Phase diagram as a function of temperature and magnetization. Solid lines running left-right show cuts at constant magnetic field $h$ taken from simulations. The coexistence regions associated with first order phase transitions are coloured white. Thick purple dashed lines show phase boundaries obtained through a low-T expansion. Further increasing anisotropy leads to suppression of supersolid phases.

resulting in a first-order plateau-plateau transition for $h=6\left(J_{1}-3 J_{2}\right)=3.3$, a value which holds up to the Ising limit $\frac{46}{6}$.

The points where both the collinear-supersolid and the plateau-plateau transitions terminate on the $\mathbb{Z}_{3}$ transition line are now well separated. They occur approximately at the same field $h \approx 1.85$ but at clearly different temperatures, $T \approx 0.19$ and $T \approx 0.34$ respectively. Hence this implies a direct transition between the stripe phase and the $m=1 / 3$ plateau as a function of field. We thus interpret the aforementioned closeness of these two points at $D=0.5$ as purely accidental for that specific value anisotropy, and not a robust feature of the model.

The previously identified points where the magnetisation jumps disappear at the plateau-stripe critical point and at both plateau-plateau and paramagnet-plateau transitions are still clearly visible. We have checked that these features hold for several values of intermediate $t D$ and thus can be said to be characteristic of the $m=1 / 2$ plateau phase.

A relevant difference is that the $3: 1$ canted phase has now become separate from the parent $m=1 / 2$ plateau for all temperatures in the magnetisation-temperature phase diagram. The increased anisotropy favours the collinear state, driving the transition first order, instead of the symmetry-allowed second-order transition observed before. At $T=0$ the mean-field transition field is $h=6.57$, which is lower than the field predicted by the closing of the spin-wave gap Eq. 222, $h_{c}(D=0.675)=6.72$. This crossover to a first-order transition happens at $D \approx 0.525$.

The simulation results agree with this interpretation, showing for all temperatures the hallmarks of a first order transition, jumps in order parameters and magnetisation and double-peaked energy distribution [Fig. 27]. The nature of the paramagnet-canted transition is unchanged, displaying a critical endpoint at $h \approx 7.2$ and $T \approx 0.06$ where fluctuations at higher temperatures drive the transition first-order. 

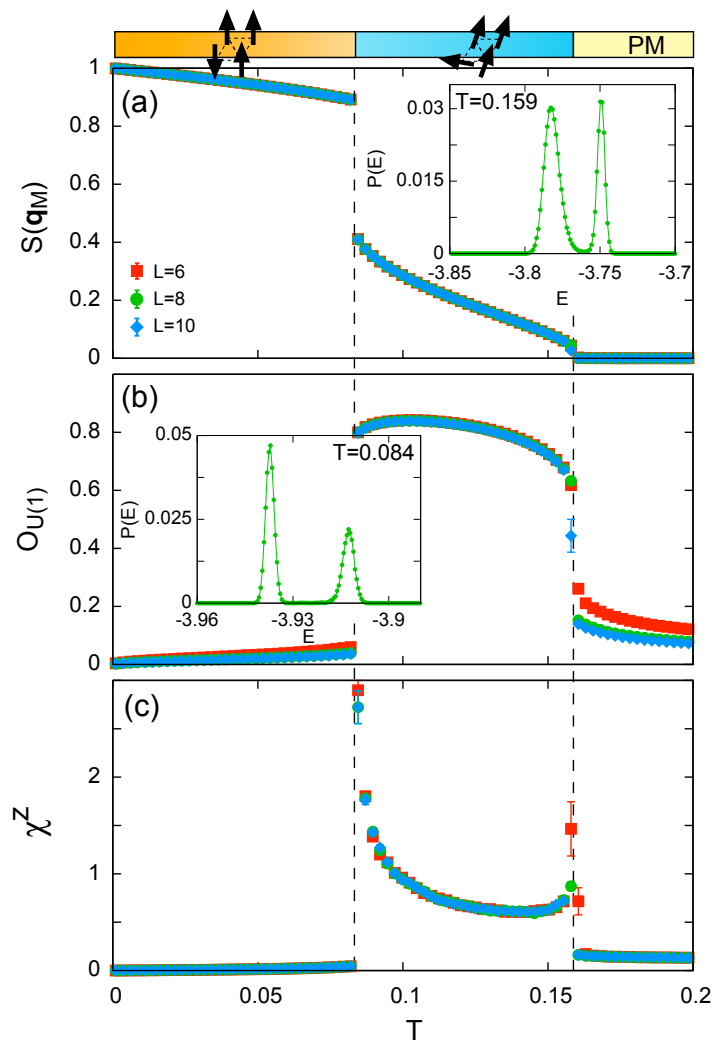

FIG. 27: (color online) Double first-order transition at $D=0.675, h=6.4$ from the paramagnet into $3: 1$ canted state, and from 3:1 canted state into the $m=1 / 2$ plateau. Temperature dependence of the structure factor $\mathcal{S}^{z z}\left(\mathbf{q}_{\mathrm{M}}\right)$ (a), $U(1)$ order parameter (b) and magnetic susceptibility (c). The first-order character of both transitions is clear in the order parameter jumps and double-peaked energy distributions close to the critical temperatures in insets to (a) and (b). The lower transition is continuous for smaller $D$, e.g. $D=0.25$ [Fig. 18 .

\section{STRONG ANISOTROPY, $D=1.5$}

Increasing the anisotropy strength eventually leads to the suppression of all non-collinear phases. At $T=0$, the supersolid state is squeezed out of existence at $D>0.9$ for $J_{2}=0.15$ by the collinear phases which surround it. For anisotropy values of $D \gtrsim 1.15$ the $3: 1$ canted phase disappears and there is only a direct plateau-saturation transition, i.e. a magnetisation jump from $m=1 / 2$ to $m=1$ with increasing field. We thus focus on $D=1.5$ as a representative value.

The ordered observed phases are those collinear phases which can be accommodated in a three- or a four-site unit cell, i.e. the collinear stripe phase at low field and then the $m=1 / 3$ - and $m=1 / 2$ magnetisation plateaux. At $T=0$ the transition fields are independent of the anisotropy value and agree with the established results for the Ising model. All phase transitions are first order for all temperatures and fields.

Nevertheless, even in this strong-anisotropy regime the features above identified previously as robust character- istics of the model are still clearly visible. These include the persistence of the first-order transition between the stripe-collinear and $m=1 / 2$ phases, and the points with $\Delta m=0$ in both the paramagnet-plateau and plateauplateau transitions.

\section{THE ISING LIMIT, $D=\infty$}

It is instructive to compare the strong anisotropy results with the pure Ising limit, $D \rightarrow \infty$, previously investigated at $T=0$ [Ref. 46]. The most obvious difference is that in the Ising limit the $m=1 / 3$ plateau is now directly connected to the paramagnetic state. The two values of field where the magnetisation jump at the first-order paramagnet- $\mathrm{m}=1 / 2$ plateau transition are still present, but one of them now arises where both plateaux and the paramagnet meet. The inner transition fields all show very little dependence with temperature, indicating the validity of the mean-field picture and that all three phases possess roughly the same amount of entropy. As a final comment, we observe that the zero field Néel temperature is a monotonically function of anisotropy. Empirically, this can be written as $T_{N}(D)=T_{N}(\infty)-\frac{1.81(1)}{2.21(4)+D}$, until saturating at $T_{N}(\infty)=1.127(3)$ in the Ising limit.

\section{DISCUSSION OF RESULTS}

TABLE I: Catalogue of phases and associated phase diagrams for different values of $D$.

\begin{tabular}{|c|c|c|c|c|c|c|c|c|}
\hline \multirow{3}{*}{ phase } & \multirow{3}{*}{ Fig. } & \multicolumn{7}{|c|}{$D$} \\
\hline & & 0 & 0.02 & 0.25 & 0.5 & 0.675 & 1.5 & $\infty$ \\
\hline & & \begin{tabular}{|l|l} 
Fig & 6 \\
\end{tabular} & \begin{tabular}{|l|l|} 
Fig & 10 \\
\end{tabular} & Fig 13 & \begin{tabular}{|l|l||} 
Fig & 24 \\
\end{tabular} & Fig 26 & \begin{tabular}{|l|l|l|} 
Fig & 28 \\
\end{tabular} & \begin{tabular}{|l|l|} 
Fig & 29 \\
\end{tabular} \\
\hline collinear stripe & $2(\mathrm{a})$ & $\checkmark$ & $\checkmark$ & $\checkmark$ & $\checkmark$ & $\checkmark$ & $\checkmark$ & $\checkmark$ \\
\hline spin flop & $2(b)$ & $\checkmark$ & $\checkmark$ & $\times$ & $\times$ & $\times$ & $\times$ & $\times$ \\
\hline supersolid & $2(\mathrm{c})$ & $x$ & $\checkmark$ & $\checkmark$ & $\checkmark$ & $\checkmark$ & $x$ & $x$ \\
\hline$m=1 / 3$ plateau & $2(\mathrm{~d})$ & $x$ & $x$ & $\times$ & $\checkmark$ & $\checkmark$ & $\checkmark$ & $\checkmark$ \\
\hline $2: 1: 1$ canted & $2(\mathrm{e})$ & $x$ & $\checkmark$ & $\checkmark$ & $\checkmark$ & $x$ & $x$ & $x$ \\
\hline$m=1 / 2$ plateau & $2(\mathrm{f})$ & $\checkmark$ & $\checkmark$ & $\checkmark$ & $\checkmark$ & $\checkmark$ & $\checkmark$ & $\checkmark$ \\
\hline $3: 1$ canted & $2(\mathrm{~g})$ & $\checkmark$ & $\checkmark$ & $\checkmark$ & $\checkmark$ & $\checkmark$ & $x$ & $x$ \\
\hline
\end{tabular}

In this paper, we have explored the magnetic-field properties of a frustrated easy-axis Heisenberg model, originally introduced to explain the magnetic properties of the hexagonal delafossite $2 \mathrm{H}-\mathrm{AgNiO}_{2}$ [42. We have used extensive Monte Carlo simulations, combined with Landau theory and spin-wave analysis, to obtain a set of magnetic phase diagrams for a set of values of singleion anisotropy, ranging from the Heisenberg $[D=0]$ to the Ising $[D=\infty]$ limits. The model exhibits a spectacularly rich set of phase diagrams, with a wide variety of competing collinear and non-collinear states, some of which are magnetic supersolids in the sense of Matsuda and 

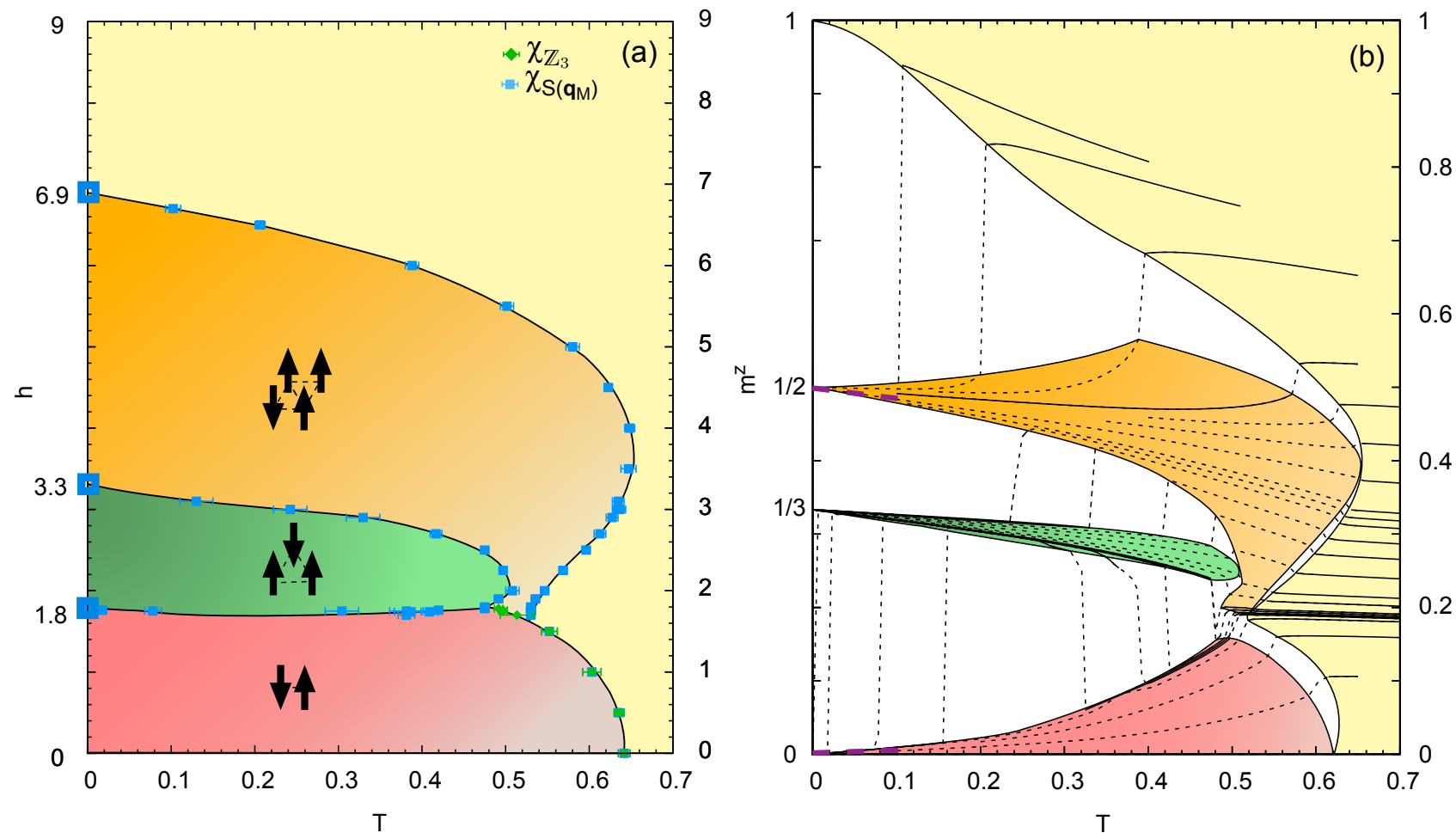

FIG. 28: (color online) (a) Magnetic phases of a layered triangular-lattice antiferromagnet with $J_{1}=1, J_{2}=0.15, J_{\perp}=-0.15$ and strong easy-axis anisotropy $D=1.5$. (a) Phase diagram as a function of temperature and magnetic field. Open symbols on the h-axis show transitions obtained in mean-field theory. Phase boundaries at finite temperature are obtained from Monte Carlo simulation for a cluster of $24 \times 24 \times 8$ spins, and determined by peaks in the relevant order parameter susceptibilities. All phase transitions are first-order. (b) Phase diagram as a function of temperature and magnetization. Solid lines running left-right show cuts at constant magnetic field $h$ taken from simulations. The coexistence regions associated with first order phase transitions are coloured white. Thick purple dashed lines show magnetisation curves obtained through a low-T expansion. At high anisotropy non-collinear phases have disappeared and Ising-like physics is recovered.

Tsuneto or Liu and Fisher. A catalogue of these phases, together with the values of $D$ for which they occur, is given in Table I.

The key to understanding the properties of the model for small values of anisotropy lies in the Heisenberg limit, $D \equiv 0$ [Fig. 6. Here the system breaks up into two, decoupled, sublattices [Fig 7], and the ordered states seen at finite temperature are selected by considerations of entropy, rather than energy. However the difference in entropy between these phases is very small, and so any finite magnetic anisotropy can stabilize new phases at low temperature, as observed for $D=0.02$ [Fig. 10. These new phases include the collinear stripe state observed in $\mathrm{AgNiO}_{2}$ [39, and the novel magnetic supersolid [Fig 2(c)] introduced in [41].

That such a supersolid should be stabilized by easy-axis anisotropy might at first seem surprising, since easy-axis anisotropy naturally suppresses the in-plane magnetization which lends a magnetic supersolid its "superfluid" character. In this case, however, the supersolid is driven by the balance of competing exchange interactions and anisotropy, and not by any delicate order-from-disorder effect. This energetic origin of the supersolid makes it very robust - it is present at low temperatures for a wide range of values of anisotropy $0<D \lesssim 0.9$, as illustrated in the phase diagrams for $D=0.02$ [Fig. 10, $D=0.25$ [Fig. 13], $D=0.5$ [Fig. 24 and $D=0.675$ [Fig. 26].

The transition from the collinear "stripe" state [Fig. 2(a)] into the magnetic supersolid, as a function of magnetic field, can be viewed as the condensation of spin wave excitations with finite momentum. This sets the new state apart from other, known examples of magnetic supersolids, where the mode which condenses has zero momentum. As a consequence the supersolid does not simply interpolate between collinear stripe (solid) and spin-flop (superfluid) states, or terminate in a tetracritical point, as envisaged by Liu and Fisher ${ }^{27}$.

Instead, at higher temperatures, the supersolid is "squeezed out" of the magnetic phase diagram by competing phases with higher entropy - the collinear "stripe" phase from which it descends, its canted "spinflop" analogue $[D=0.02$; Fig. 10$]$ and, at higher values of $D$, collinear $m=1 / 3[D=0.25$; Fig. 13 and $D=0.5$; Fig. 24 and $m=1 / 2$ magnetization plateaux $[D=0.675$; Fig. 26. We anticipate that increasing the secondneighbour interaction $J_{2}$ (fixed in these simulations at $J_{2}=0.15 J_{1}$ ), will make the magnetic supersolid more robust at higher temperatures. 

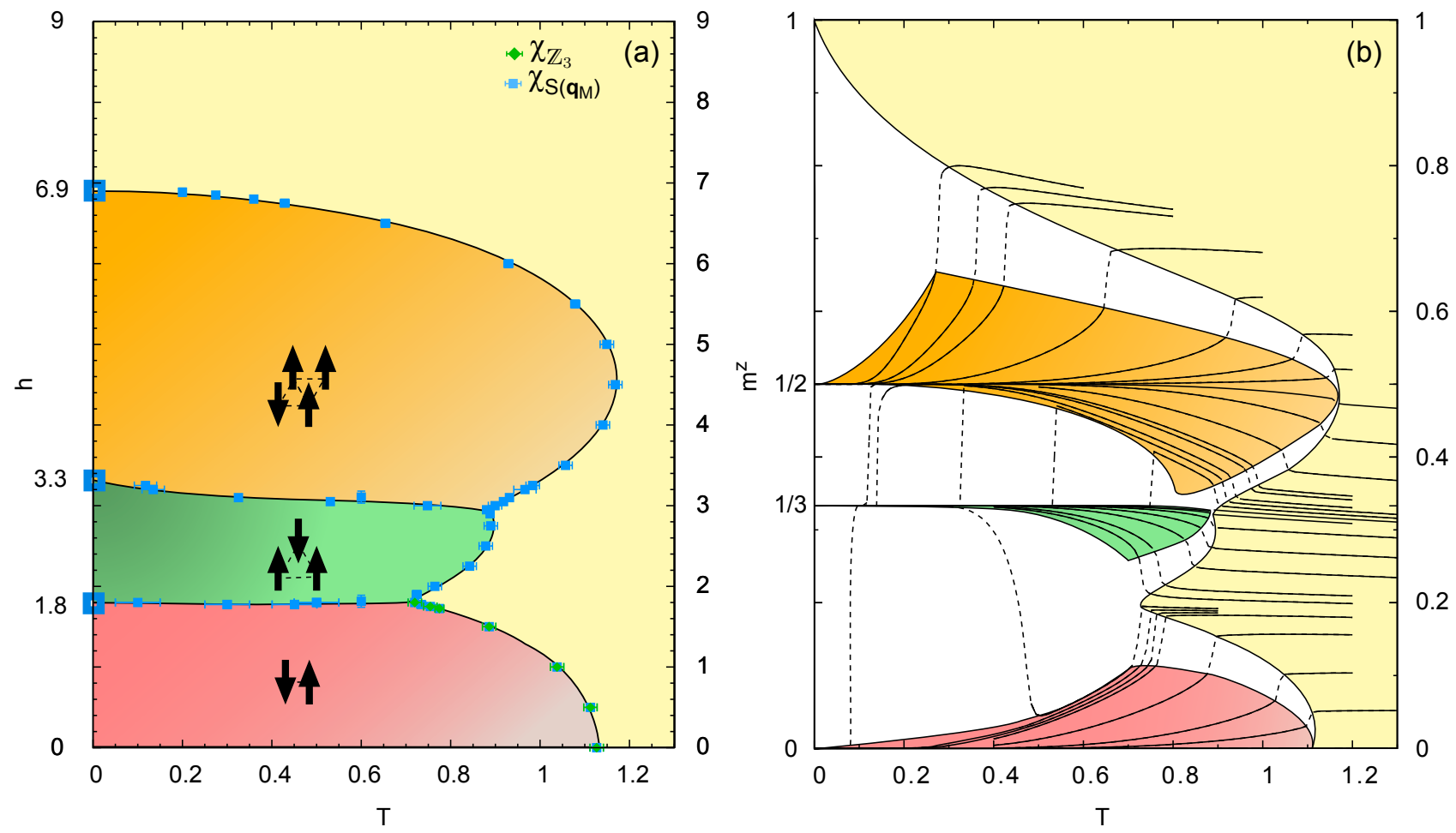

FIG. 29: (color online) (a) Magnetic phases of a layered triangular-lattice Ising antiferromagnet with $J_{1}=1, J_{2}=0.15, J_{\perp}=-0.15$. (a) Phase diagram as a function of temperature and magnetic field. Open symbols on the h-axis show transitions obtained in mean-field theory. Phase boundaries at finite temperature are obtained from Monte Carlo simulation for a cluster of $24 \times 24 \times 8$ spins, and determined by peaks in the relevant order parameter susceptibilities. All phase transitions are first-order. (b) Phase diagram as a function of temperature and magnetization. Solid lines running left-right show cuts at constant magnetic field $h$ taken from simulations. The coexistence regions associated with first order phase transitions are coloured white.

A similar story is repeated with the two other magnetic supersolid phases found at higher values of magnetic fields : an unusual 2:1:1 canted state [Fig. 2(e)], and a $3: 1$ canted state [Fig. 2(g)]. The 2:1:1 canted state interpolates between the magnetic supersolid and a collinear $m=1 / 2$ plateau. Like the supersolid, it owes its stability to a balance of competing interactions, and is displaced at high temperatures by the collinear $m=1 / 2$ plateau, which has superior entropy. It not only exhibits a substantial staggered magnetization in the $S^{x}-S^{y}$ plane, but also a finite (if small) uniform magnetization $m_{\perp}$, perpendicular to the magnetic field. In turn, the $3: 1$ canted phase interpolates between the collinear $m=1 / 2$ plateau and saturation. Like the 2:1:1 canted phase it exhibits a small magnetization $m_{\perp}$ in the $S^{x}-S^{y}$ plane. It is the only supersolid phase present for $D=0$ (where $m_{\perp} \equiv 0$ ), [Fig. 6], and the only supersolid phase favoured by considerations of entropy alone. For this reason it is also the only supersolid connected to the paramagnetic phase at high temperatures.

The phase transitions which link these different phases also evolve as a function of $D$. Each of the canted phases is connected to neighbouring collinear phases by a soft spin-wave mode within the collinear state, and so these transitions can be continuous. However phase transitions at high temperatures are generically first order, and be- come more strongly so as anisotropy is increased, as observed by e.g. comparing the phase diagrams for $D=0.5$ [Fig. 24], $D=0.675$ [Fig. 26] and $D=1.5$ [Fig. 28]. This trend is seem most clearly in phase diagrams plotted as a function of temperature and magnetization, which begin to exhibit large regions of phase coexistence as $D$ is increased.

The anaysis in this paper clarifies the origin of the novel magnetic supersolid introduced in 41, explores its unusual properties at finite temperature, and confirms its robustness against other competing phases for a wide range of parameters. However a number of interesting questions remain. One of these is, how would the system respond for magnetic field not aligned with the easy axis? An obvious limiting case is a field perpendicular to the easy axis. In this case, the two sublattices of the collinear stripe phase can respond to field simply by canting, and (zero temperature) mean-field calculation indicate that a canted stripe phase interpolates to saturation for all finite $D$. The second obvious limit is that of a field at a small angle to the easy axis. In this case, the states described in this paper will generally survive, but the symmetries they break will be modified by the presence of a component of magnetic field in the $S^{x}-S^{y}$ plane. This in turn will lead to a modification of the phase transitions between them. The limit of small angle is also of relevance to 
experiments which measure magnetization through magnetic torque, discussed in the context of $\mathrm{AgNiO}_{2}$ above. Away from either of these limits, for intermediate angle, the story is not so simple - the balance of energy and entropy can favour many different states. This remains an interesting topic for future study.

Another interesting open question is the study of the quantum mechanical effects in this spin model. The succession of continuous phase transitions observed in our model for moderate values of anisotropy, raises the intriguing possibility that a quantum magnet like $\mathrm{AgNiO}_{2}$ could exhibit a series of quantum phase transitions as a function of magnetic field. Concentrating on the supersolid phases, two-dimensional quantum models with broadly similar Hamiltonians support supersolids both at $\mathrm{T}=0$ [Ref. 33 35] and finite temperature $36 / 54$. However, recent calculations suggest that the supersolid phase in the original three-dimensional model considered by Liu and Fisher may not survive quantum fluctuations 55 . Each case therefore needs to be considered on its own merits. One simple, phenomenological, way to incorporate quantum fluctuations is through the addition an effective biquadratic interaction term, mimicking the selection of collinear states by quantum fluctuations 56 . We have checked within classical Monte Carlo simulations that such a term does not change the nature of the transition into the supersolid phase. On the strength of this, and on general grounds, we anticipate that the supersolid introduced in 41] will prove robust against quantum effects. However this remains to be tested.

\section{APPLICATION TO EXPERIMENT}

The advent of high field facilities offering static fields of up to 45T, and pulsed fields of up to $600 \mathrm{~T}$, has made it possible to explore the high-field properties of a wide range of magnets for the first time. Frustrated magnets are foremost among these, with model systems like $\mathrm{TlCuCl}_{3}$ providing a perfect opportunity to study how the Bose-Einstein condensation of magnons gives rise to a magnetic superfluid [Ref. 3]. Generically, these systems exhibit some degree of magnetic anisotropy, either at the level of a single ion, or in their exchange interactions.

Triangular lattice antiferromagnets are no exception. Examples with weak easy-axis anisotropy (relative to exchange interactions) include the quasi-two dimensional halides $\mathrm{VBr}_{2}$ and $\mathrm{VCl}_{2} \sqrt{57 / 58}$, which contain antiferromagnetically coupled spin- $3 / 2 \quad \mathrm{~V}^{2+}$ ions on a triangular lattice. The insulating oxide $\mathrm{Rb}_{4} \mathrm{Mn}\left(\mathrm{MoO}_{4}\right)_{3} 59$ and the multiferroic material $\mathrm{RbFe}\left(\mathrm{MoO}_{4}\right)_{2} 60$, are well-described by a spin-5/2 nearest-neighbour Heisenberg antiferromagnet on a triangular lattice with moderate easy-axis anisotropy $\left(\mathrm{Rb}_{4} \mathrm{Mn}\left(\mathrm{MoO}_{4}\right)_{3}\right)$, or easyplane anisotropy $\left(\mathrm{RbFe}\left(\mathrm{MoO}_{4}\right)_{2}\right)$. The multiferroic material $\mathrm{KFe}\left(\mathrm{MoO}_{4}\right)_{2}$ has similar underlying chemistry to $\mathrm{RbFe}\left(\mathrm{MoO}_{4}\right)_{2}$, but a distortion of the triangular lattice leads to a somewhat more complex phenomenology $\sqrt{63}$.
Much stronger single-ion anisotropy, of order of the exchange interactions, is found in the hexagonal $\mathrm{Ni}$ halides $\mathrm{CsNiCl}_{3}, \mathrm{CsNiBr}_{3}{ }^{64}$ and in the $\mathrm{Cr}$ oxide $\mathrm{LiCrO}_{2}{ }^{65}$, where triangularly coordinated $S=3 / 2$ spins order on the magnetic easy-axis. The insulating oxide

Further examples are found in the delafossite family, including $S=5 / 2 \mathrm{CuFeO}_{2} \sqrt{66}, S=3 / 2 \mathrm{PdCrO}_{2} \frac{67}{6}$ and $S=1 \mathrm{AgNiO}_{2}{ }^{3942}$ with easy-axis anisotropy, and $S=3 / 2$

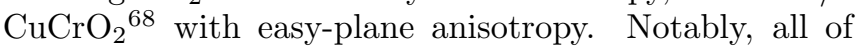
these delafossite materials require a frustrated model such as Eq. (1), with second (or further) neighbour interactions, to accurately describe their magnetic properties. Here we have concentrate on $\mathrm{AgNiO}_{2}$, where powder neutron scattering studies provide clear evidence for a collinear, stripe ground state in zero magnetic field 39 , as would be expected for the frustrated, easy-axis model considered in this paper with moderate $J_{2}$ and $D$.

At present, single crystals of $\mathrm{AgNiO}_{2}$ are too small for inelastic neutron scattering experiments to be performed on them. However angle-integrated spin-wave spectra can be measured in powder samples. These show clear evidence of a spin-gap, and spectra are welldescribed by the present model [Eq. (1)], with parameters $J_{1}=1.32 \mathrm{meV}, J_{2}=0.20 \mathrm{meV}, D=1.78 \mathrm{meV}, J_{\perp}=-0.14$ meV 42 . For spin $S=1$, the effective value of $D$ seen in quantum spin wave spectra is renormalized by a factor two relative to its classical value ${ }^{42}$, and so comparison should be made with classical Monte Carlo simulation results for $D=0.675 \times J_{1}$. Thus, under the (strong) assumption that this simple spin model provides an adequate description of $\mathrm{AgNiO}_{2}$ in high magnetic field, the field-temperature phase diagram for $\mathrm{AgNiO}_{2}$ should be of the form shown in Fig. 26 [ $D=0.675]$. Given the simplifications inherent in using any spin model to describe a metal, the critical fields and transitions temperatures predicted here should be approached with some caution. are unlikely to be quantitatively correct. However our Monte Carlo simulation results should provide a reasonable first guide to the different phases occuring in $\mathrm{AgNiO}_{2}$ in magnetic field, and the nature of the phase transitions between them.

The actual phase transitions which occur in $\mathrm{AgNiO}_{2}$ for fields of up to 40T, have been studied through meaurements of magnetic torque $\vec{\tau}=\mathbf{m} \times \mathbf{h}$, heat capacity and electrical transport $\frac{40}{4}$. These experiments indicate that a continuous (or very weakly first order) phase transition out of the collinear stripe state occurs at about $12.5 \mathrm{~T}$ (at a base temperature of $1.5 \mathrm{~K}$ ). This phase transition is accompanied by a weak anomaly in the specific heat. And, crucially, it occurs at a critical field which increases steadily with temperature, indicating that the collinear stripe phase has a higher entropy than its successor. These are exactly the characteristics of the phase transition from the collinear stripe phase into the supersolid for the frustrated easy-axis model Eq. (1).

In Ref. [41] we therefore proposed that a novel magnetic supersolid is realised in $\mathrm{AgNiO}_{2}$ for magnetic fields greater than $12.5 T$. We further suggested that torque 
was a good tool for distinguishing phase transitions in easy-axis magnets, exhibiting changes in sign as well as in magnitude, and made explicit predictions for the torque signature of the proposed magnetic supersolid 41 . It is also interesting to note that torque clearly distinguishes those phases, such as the 2:1:1 canted phase and the 3:1 canted phase above, which have a finite magnetization $m_{\perp}$ in the $S^{x}-S^{y}$ plane.

The results of this paper confirm that the scenario presented in 41] is robust across a wide range of parameter space, and does not require any special assumptions or fine-tuning of the model. In this context it would be interesting to look for evidence of the spin gap closing at the putative supersolid transition in $\mathrm{AgNiO}_{2}$, either from powder neutron scattering, or NMR relaxation rates. However it is important also to remember this model is a gross simplification of the physics of $\mathrm{AgNiO}_{2}$, which contains itinerant electrons as well as local moments. The development of a more realistic model for $\mathrm{AgNiO}_{2}$, which takes these itinerant electrons into account, remains an important avenue for future study.

\section{CONCLUSIONS}

In this paper we have studied a realistic, threedimensional spin model motivated by the hexagonal delafossite $2 \mathrm{H}-\mathrm{AgNiO}_{2}$. We have obtained the full magnetic phase diagram of this model as a function of temperature and magnetic field for values of easy-axis anisotropy $D$ ranging from the Heisenberg $(D=0)$ to the Ising $(D=\infty)$ limits. We uncovered a rich variety of different magnetic phases, including several phases which are magnetic supersolids (in the sense of Matsuda and Tstuneto or Liu and Fisher), one of which may already have been observed in $\mathrm{AgNiO}_{2}$. We . We explored how this particular supersolid, first introduced in [41, arises through the closing of a gap in the spin-wave spectrum, and how it competes with neighbouring phases as the easy-axis anisotropy is increased. This novel phase was shown to have qualitatively different finite-temperature properties from any previously studied magnetic supersolid, and to be remarkably robust against changes in parameters. These results suggest that magnetic supersolids in frustrated systems can have a richer phonomenolgy, and be far more robust, than previously supposed.

\section{Acknowledgments}

The authors thank Tony Carrington, Andrey Chubukov, Amalia and Radu Coldea, Andreas Läuchli, Yukitoshi Motome, Wolfgang Selke and Mike Zhitomirsky for helpful comments on this work. Numerical simulations made use of the Advanced Computing Research Centre, University of Bristol. This work was supported by FCT Grant No. SFRH/BD/27862/2006 and EPSRC Grants No. EP/C539974/1 and EP/G031460/1.

\section{Appendix A: Calculation of spin stiffness}

Spin stiffness is defined as a generalised elasticity coefficient representing the free-energy cost of applying a twist to the boundary conditions with gradient $\delta \phi$ along a given direction ê. Since there is a direct mapping between spin stiffness and superfluid density 69 , a phase with nonvanishing $\rho_{S}$ is said to have superfluid character. In finite magnetic field it is sufficient to consider twists around $S^{z}$, i.e in the $S^{x}-S^{y}$ plane. For a single pair of spins we write

$$
\mathbf{S}_{i} \cdot \mathbf{S}_{j}^{\prime}=\cos \left(\phi_{i}-\phi_{j}+\delta \phi . \hat{\mathbf{e}} .\left(\mathbf{r}_{i}-\mathbf{r}_{j}\right)\right),
$$

where each spin is expressed in polar coordinates

$$
\mathbf{S}_{i}=\left(\cos \phi_{i} \sin \theta_{i}, \sin \phi_{i} \sin \theta_{i}, \cos \theta_{i}\right) \text {. }
$$

Spin stiffness is then given by the second derivative of the free energy with respect to the twisting angle $\delta \phi$

$\rho_{s}[\hat{\mathbf{e}}]=\left.\frac{\partial^{2} \mathcal{F}}{\partial(\delta \phi)^{2}}\right|_{\delta \phi=0}=\left.\left\langle\frac{\partial^{2} \mathcal{H}}{\partial(\delta \phi)^{2}}\right\rangle\right|_{\delta \phi=0}-\left.\frac{1}{T}\left\langle\left(\frac{\partial \mathcal{H}}{\partial(\delta \phi)}\right)^{2}\right\rangle\right|_{\delta \phi=0}$.

Applying Eq. A3 to both first- and second-neighbour interactions and normalising per unit area (i.e. per spin), spin stiffness reads $\underline{69 / 70}$

$$
\begin{gathered}
\rho_{s}(\hat{\mathbf{e}})=-\frac{2}{\sqrt{3} N}\left\langle J_{1} \sum_{\langle i, j\rangle_{1}}\left(\hat{\mathbf{e}} \cdot \mathbf{r}_{i j}\right)^{2} \cdot \mathbf{S}_{i}^{\perp} \cdot \mathbf{S}_{j}^{\perp}+J_{2} \sum_{\langle i, j\rangle_{2}}\left(\hat{\mathbf{e}} \cdot \mathbf{r}_{i j}\right)^{2} \cdot \mathbf{S}_{i}^{\perp} \cdot \mathbf{S}_{j}^{\perp}\right\rangle \\
-\frac{2}{\sqrt{3} N T}\left\langle\left\{J_{1} \sum_{\langle i, j\rangle_{1}}\left(\hat{\mathbf{e}} \cdot \mathbf{r}_{i j}\right) \cdot \mathbf{S}_{i}^{\perp} \times \mathbf{S}_{j}^{\perp}+J_{2} \sum_{\langle i, j\rangle_{2}}\left(\hat{\mathbf{e}} \cdot \mathbf{r}_{i j}\right) \cdot \mathbf{S}_{i}^{\perp} \times \mathbf{S}_{j}^{\perp}\right\}^{2}\right\rangle,
\end{gathered}
$$

where $\mathbf{r}_{i j}=\mathbf{r}_{i}-\mathbf{r}_{j}$. Since the parallel tempering method restores the full lattice symmetries, we average $\rho_{S}$ over the three symmetric directions in the lattice $\hat{\mathbf{e}}=\left(\hat{\mathbf{e}}_{x}, \hat{\mathbf{e}}_{y}\right)=\{(1,0),(1 / 2, \sqrt{3} / 2),(-1 / 2, \sqrt{3} / 2)\}$.

\section{Appendix B: Low temperature expansion and Landau theory for supersolid transition}

The low-temperature excitations of a classical spin model of the form Eq. (1) are frozen spin waves, in contrast with the dynamic spin waves found in quantum magnets. These excitations have a dispersion which can be calculated through an expansion of each spin fluctuations around its preferred $T=0$ configuration. In the sufficiently generic case of two spins in a common plane canted by an angle $2 \theta$, the Heisenberg interaction is approximated by

$$
\begin{aligned}
\mathbf{S}_{i} \cdot \mathbf{S}_{j} \approx & \cos 2 \theta-\frac{1}{2}\left(x_{i}^{2}+x_{j}^{2}+y_{i}^{2}+y_{j}^{2}\right) \cos 2 \theta \\
& +x_{i} x_{j} \cos 2 \theta+y_{i} y_{j},
\end{aligned}
$$


where $x_{i}$ and $y_{i}$ denote small fluctuations about the ordered state in the local $S_{i}^{x}$ and $S_{i}^{y}$ directions respectively. The rotation into a common frame is arbitrarily chosen to be performed around the $S^{y}$ direction.

The resulting low-temperature Hamiltonian is solved by Fourier transform, with two spin-wave modes $\epsilon_{\alpha}(\mathbf{k})$ per $n$ sublattice, i.e.

$$
\mathcal{H}=\mathcal{H}_{0}+\frac{1}{2} \sum_{\alpha}^{2 n} \sum_{\mathbf{k} \in M_{B Z}}^{N / n} \epsilon_{\alpha}(\mathbf{k}) x_{\alpha_{\mathbf{k}}} x_{\alpha_{-\mathbf{k}}} .
$$

We now illustrate this procedure for the supersolid state with four different sublattices : while two are collinear with $S^{z}$ in the positive direction the other two are canted by an angle of $\theta$ from $S^{z}$ in opposite directions. After writing down the interactions between sublattices in matrix form, the spin-wave dispersion modes can be obtained by diagonalisation of

$$
\begin{gathered}
M_{\mathbf{k}}^{x}=2\left[\begin{array}{cccc}
V_{\mathbf{k}} & X_{\mathbf{k}} & Y_{\mathbf{k}} \cdot c_{\theta} & Z_{\mathbf{k}} \cdot c_{\theta} \\
X_{\mathbf{k}} & V_{\mathbf{k}} & Z_{\mathbf{k}} \cdot c_{\theta} & Y_{\mathbf{k}} \cdot c_{\theta} \\
Y_{\mathbf{k}} \cdot c_{\theta} & Z_{\mathbf{k}} \cdot c_{\theta} & W_{\mathbf{k}}+D \cdot c_{2 \theta} & X_{\mathbf{k}} \cdot c_{2 \theta} \\
Z_{\mathbf{k}} \cdot c_{\theta} & Y_{\mathbf{k}} \cdot c_{\theta} & X_{\mathbf{k}} \cdot c_{2 \theta} & W_{\mathbf{k}}+D \cdot c_{2 \theta}
\end{array}\right], \\
M_{\mathbf{k}}^{y}=2\left[\begin{array}{cccc}
V_{\mathbf{k}} & X_{\mathbf{k}} & Y_{\mathbf{k}} & Z_{\mathbf{k}} \\
X_{\mathbf{k}} & V_{\mathbf{k}} & Z_{\mathbf{k}} & Y_{\mathbf{k}} \\
Y_{\mathbf{k}} & Z_{\mathbf{k}} & W_{\mathbf{k}}+D \cdot c_{\theta}^{2} & X_{\mathbf{k}} \\
Z_{\mathbf{k}} & Y_{\mathbf{k}} & X_{\mathbf{k}} & W_{\mathbf{k}}+D \cdot c_{\theta}^{2}
\end{array}\right]
\end{gathered}
$$

where $c_{\theta}=\cos \theta$ and the other coefficients are given by

$$
\begin{aligned}
& V_{\mathbf{k}}=\left(J_{1}+J_{2}\right)\left(2 c_{\theta}-1\right)+J_{\perp}\left(\gamma_{z}(\mathbf{k})-1\right)+D+\frac{h}{2} \\
& W_{\mathbf{k}}=\left(J_{1}+J_{2}\right)\left(2 c_{\theta}-c_{2 \theta}\right)+J_{\perp}\left(\gamma_{z}(\mathbf{k})-1\right)-\frac{h}{2} c_{\theta} \\
& X_{\mathbf{k}}=J_{1} \gamma_{1}^{A B}(\mathbf{k})+J_{2} \gamma_{2}^{A B}(\mathbf{k}) \\
& Y_{\mathbf{k}}=-J_{1} \gamma_{1}^{+}(\mathbf{k})-J_{2} \gamma_{2}^{-}(\mathbf{k}) \\
& Z_{\mathbf{k}}=-J_{1} \gamma_{1}^{-}(\mathbf{k})-J_{2} \gamma_{2}^{+}(\mathbf{k}) .
\end{aligned}
$$

The lattice structure factors are $\gamma_{1}^{A B}(\mathbf{k})=\cos k_{x}, \quad \gamma_{2}^{A B}(\mathbf{k})=\cos \sqrt{3} k_{y}, \quad \gamma_{z}(\mathbf{k})=\cos k_{z}$, $\gamma_{1}^{ \pm}(\mathbf{k})=\cos \left(\left(k_{x} \pm \sqrt{3} k_{y}\right) / 2\right) \quad$ and $\quad \gamma_{2}^{ \pm}(\mathbf{k})=\cos \left(\left(3 k_{x} \pm\right.\right.$ $\left.\sqrt{3} k_{y}\right) / 2$ ). The four-sublattice structure results in 8 dispersing modes $\epsilon(\mathbf{k})$. The thermodynamics at low-T are accessed via the free energy,

$$
\frac{\mathcal{F}}{N}=\frac{E_{0}}{N}-T \ln T+\frac{1}{2} \frac{T}{N} \sum_{\alpha}^{2 n} \sum_{\mathbf{k} \in M_{B Z}}^{N / n} \ln \epsilon_{\alpha}(\mathbf{k})+O\left(T^{2}\right) .
$$

The free energy of the supersolid phase near its phase transition can be obtained as a Landau expansion in powers of the order parameter measuring the $U(1)$ broken symmetry

$$
\mathcal{F} \approx \mathcal{F}_{0} \frac{a}{2}\left|O_{U(1)}\right|^{2}+\frac{b}{4}\left|O_{U(1)}\right|^{4}+\ldots .
$$

The $U(1)$ order parameter [Eq. (5)] is by construction proportional to the canting angle $\theta$, as represented in the cartoon Fig. 2(c), and therefore near the transition

$$
O_{U(1)}=2 \sin \theta \sim \theta
$$

The coefficient $a$ collects the quadratic contributions from the spin wave modes

$$
\begin{aligned}
a & =h_{\mathrm{SSD}}(T)-h \\
h_{\mathrm{SSD}}(T) & =2 D+\left.2 T \frac{\partial^{2}}{\partial \theta^{2}}\left\{\frac{1}{2 N} \sum_{\alpha, \mathbf{k}} \ln \epsilon_{\alpha}(\mathbf{k})\right\}\right|_{\theta=0},
\end{aligned}
$$

where the critical field is calculated near $T=0$. The prefactor for the temperature term is obtained by numerically integrating the derivatives of the eight spinwave branches over the four-sublattice magnetic Brillouin Zone, corresponding to the finite lattice used in simulation. The resulting critical fields are plotted as a yellow line on the (a) panel of Figs. 10,13, 24 and 26, yielding a very good agreement with simulation, even at relatively high temperatures.
1 Frustrated Spin Systems, edited by H. T. Diep, World Scientific, Singapore, (2005).

${ }^{2}$ L. Balents, Nature 464, 199 (2010).

3 T. Giamarchi, C. Ruegg, and O. Tchernyshyov, Nat. Phys. 4, 198 (2008).

4 K. Penc, N. Shannon and H. Shiba, Phys. Rev. Lett. 93, 197203 (2004).

${ }^{5}$ G. H. Wannier, Phys. Rev. 79, 357 (1950).

${ }^{6}$ P.W. Anderson, Mater. Res. Bull. 8, 153 (1973).

7 P. Fazekas and P.W. Anderson, Philos. Mag. 30, 423 (1974).

8 H. Kawamura and S. Miyashita, J. Phys. Soc. Jpn. 53, 4138 (1984).

${ }^{9}$ H. Kawamura and S. Miyashita, J Phys. Soc. Jpn. 54, 4530
(1985).

10 A.V. Chubukov and D. I. Golosov, J. Phys. Condens. Matter 3, 69 (1991).

11 T. Momoi, H. Sakamoto and K. Kubo, Phys. Rev. B 59, 9491 (1999).

12 T. Momoi, P. Sindzingre and N. Shannon, Phys. Rev. Lett. 97, 257204 (2006).

13 A. Läuchli, F. Mila and K. Penc, Phys. Rev. Lett. 97, 087205 (2006).

14 H. Tsunetsugu and M. Arikawa, J. Phys. Soc. Jpn. 75 083701, (2006).

15 Y. Shimizu, K. Miyagawa, K. Kanoda, M. Maesato, and G. Saito, Phys. Rev. Lett. 91, 107001 (2003).

16 M. Yamashita et al., Science 328, 1246-1248 (2010). 
17 K. Ishida et al., Phys. Rev. Lett. 79, 3451 (1997).

18 S. Nakatsuji, Y. Nambu, H. Tonomura, O. Sakai, S. Jonas, C. Broholm, H. Tsunetsugu, Y. Qiu and Y. Maeno, Science 309, 1114727 (2005).

19 M. Collins and O. Petrenko, Can. J. Phys. 75, 605 (1997).

20 S. Miyashita and H. Kawamura, J Phys. Soc. Jpn. 543385 (1985).

21 S. Miyashita, J. Phys. Soc. Jpn. 55, 3605 (1986).

22 Q. Sheng and C. L. Henley, J. Phys. Condens. Matter 4, 2937 (1992).

23 W. Stephan and B.W. Southern, Phys. Rev. B 61, 11514 (2000).

24 P.-É. Melchy and M. E. Zhitomirsky, Phys. Rev. B 80, 064411 (2009).

25 A. Sen, F. Wang, K. Damle, and R. Moessner, Phys. Rev. Lett. 102, 227001 (2009).

${ }^{26}$ H. Matsuda and T. Tsuneto, Sup. Prog. Theor. Phys., 46 411, (1970).

${ }^{27}$ K.S. Liu and M.E. Fisher, J. Low Temp. Phys. 10, 655 (1973).

28 G. V. Chester, Phys. Rev. A 2, 256 (1970)

29 A. J. Leggett, Phys. Rev. Lett. 25, 1543 (1970).

30 A. F.FAndreev and I. M. Lifshitz, Sov. Phys. Usp. 13670 (1971).

31 E. Kim and M.H.W. Chan, Nature 427, 225 (2004).

32 S. Balibar, Nature 464, 176 (2010).

33 S. Wessel and M. Troyer, Phys. Rev. Lett. 95, 127205 (2005).

34 D. Heidarian and K. Damle, Phys. Rev. Lett. 95, 127206 (2005).

35 R.G. Melko, A. Paramekanti, A. A. Burkov, A. Vishwanath, D. N. Sheng, and L. Balents, Phys. Rev. Lett. 95, 127207 (2005).

${ }^{36}$ M. Boninsegni and N. Prokof'ev, Phys. Rev. Lett. 95, 237204 (2005).

37 M. Holtschneider and W. Selke, Phys. Rev. B 76, 220405 (2007).

${ }^{38}$ M. Holtschneider and W. Selke, Eur. Phys. J. B 62, 147 (2008).

39 E. Wawrzyńska, R. Coldea, E.M. Wheeler, I.I. and Mazin, M.D. Johannes, T. Sörgel, M. Jansen, R.M. Ibberson, and P.G. Radaelli, Phys. Rev. Lett. 99157204 (2007); E. Wawrzyńska, R. Coldea, E.M. Wheeler, T. Sörgel, M. Jansen, R.M. Ibberson, P.G Radaelli, M.M Koza, Phys Rev B 77094439 (2008).

40 A. I. Coldea, A. Carrington, R. Coldea, L. Malone, A. F. Bangura, M. D. Johannes, I. I. Mazin, E. A. Yelland, J.G. Analytis, J. A.Perenboom,C. Jaudet, D. Vignolles, T. Sörgel and M. Jansen, preprint arXiv:0908.4169v1 (2009).

41 L. Seabra and N. Shannon, Phys. Rev. Lett. 104, 237205 (2010).

42 E.M. Wheeler, R. Coldea, E. Wawrzyńska, T. Sörgel, M. and Jansen, M.M. Koza, J. Taylor, P. Adroguer and N. Shannon, Phys. Rev. B 79, 104421 (2009).

43 J. T. Haraldsen, M. Swanson, G. Alvarez and R. S. Fishman, Phys. Rev. Lett. 102, 237204 (2009).

44 T. Takagi and M. Mekata, J. Phys. Soc. Jpn. 64, 4609 (1995).

45 B. Metcalf, Phys. Lett. A 46 325, (1974).

${ }^{46}$ K. Takasaki, I. Harada and T. Tonegawa, J. Phys. Soc.
Jpn. 55, 4423 (1986).

47 Y. Motome, K. Penc and N. Shannon, J. Magn. Magn. Mater. 300, 57 (2006).

48 N. Shannon, K. Penc and Y. Motome, Phys Rev. B 81, 184409 (2010).

49 D. Loison and H.T. Diep, Phys. Rev. B 50, 16453 (1994).

50 T. Jolicoeur, E. Dagotto, E. Gagliano and S. Bacci, Phys. Rev. B 42, 4800 (1990).

51 A.V. Chubukov and T. Jolicoeur, Phys. Rev. B 46, 11137 (1992).

52 K. Hukushima and K. Nemoto, J. Phys. Soc. Jpn. 65, 1604 (1996).

53 K. Kanki, D. Loison and K.D. Schotte, Eur. Phys. J. B 44, 309 (2005).

54 N. Laflorencie and F. Mila, Phys. Rev. Lett. 99, 027202 (2007).

55 H. T. Ueda and K. Totsuka, Phys. Rev. B 81, 054442, (2010).

56 B. E. Larson and C. L. Henley, preprint arXiv:0811.0955 (2009).

57 K. Hirakawa, H. Kadowaki, and K. Ubukoshi J. Phys. Soc. Jpn. 52, 1814 (1983).

58 H. Kadowaki, K. Ubukoshi, K. Hirokawa, J. L. Martinez and G. Shirane, J. Phys. Soc. Jpn. 56, 4027 (1987).

59 R. Ishii, S. Tanaka, K. Onuma, Y. Nambu, M. Tokunaga, T. Sakakibara, N. Kawashima, Y. Maeno, C. Broholm, D. P. Gautreaux, J. Y. Chan and S. Nakatsuji, preprint arXiv:0912.4796 1 (2009).

60 L. E. Svistov, A. I. Smirnov, L. A. Prozorova, O. A. Petrenko, L. N. Demianets, and A. Y. Shapiro, Phys. Rev. B 67, 094434 (2003).

61 L. E. Svistov, A. I. Smirnov, L. A. Prozorova, O. A. Petrenko, A. Micheler, N. Büttgen, A. Y. Shapiro, and L. N. Demianets, Phys. Rev. B 74, 024412 (2006).

62 A. I. Smirnov, H. Yashiro, S. Kimura, M. Hagiwara, Y. Narumi, K. Kindo, A. Kikkawa, K. Katsumata, A. Y. Shapiro, and L. N. Demianets, Phys. Rev. B 75, 134412 (2007).

63 A. I. Smirnov, L. E. Svistov, L. A. Prozorova, A. Zheludev, M. D. Lumsden, E. Ressouche, O. A. Petrenko, K. Nishikawa, S. Kimura, M. Hagiwara, K. Kindo, A. Y. Shapiro, and L. N. Demianets. Phys. Rev. Lett. 102, 037202 (2009).

64 M. Sano, K. Iio and K. Nagata, Phys. Rev. B 39, 97539756 (1989).

65 H. Kadowaki, H. Takei and K. Motoya, J. Phys. Condens. Matter 7, 6869 (1995).

${ }^{66}$ F. Ye, J. A. Fernandez-Baca, R. S. Fishman, Y. Ren, H. J. Kang, Y. Qiu and T. Kimura, Phys. Rev. Lett. 99, 157201 (2007).

67 H. Takatsu, H. Yoshizawa, S. Yonezawa and Y. Maeno, Phys. Rev. B 79, 104424 (2009).

68 M. Poienar, F. Damay, C. Martin, J. Robert and S. Petit, Phys. Rev. B 81, 104411 (2010).

69 T. Ohta and D. Jasnow, Phys. Rev. B 20, 139 (1979).

70 S. Teitel and C. Jayaprakash, Phys. Rev. B 27, 598 (1983).

71 Quantum corrections of order 1/S to linear spin-wave theory renormalise the anisotropy value to $D=3 / 2 J_{1}$. 